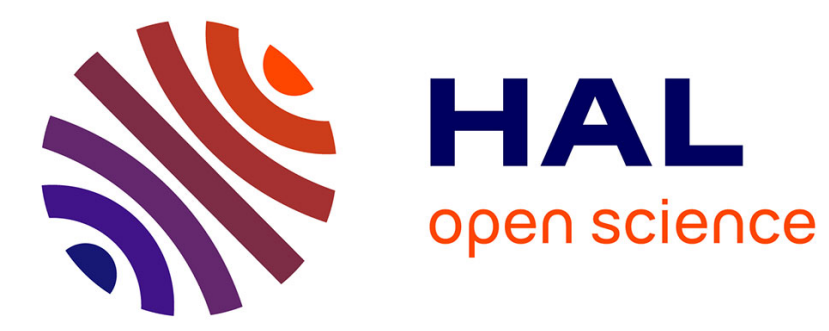

\title{
Recursive algorithms for the computation of the potential harmonic coefficients of a constant density polyhedron
}

Dimitrios Tsoulis, Olivier Jamet, Jérôme Verdun, Nicolas Gonindard

\section{- To cite this version:}

Dimitrios Tsoulis, Olivier Jamet, Jérôme Verdun, Nicolas Gonindard. Recursive algorithms for the computation of the potential harmonic coefficients of a constant density polyhedron. Journal of Geodesy, 2009, 83 (10), pp.925-942. 10.1007/s00190-009-0310-9 . hal-02612191

\section{HAL Id: hal-02612191 \\ https://hal.science/hal-02612191}

Submitted on 20 May 2020

HAL is a multi-disciplinary open access archive for the deposit and dissemination of scientific research documents, whether they are published or not. The documents may come from teaching and research institutions in France or abroad, or from public or private research centers.
L'archive ouverte pluridisciplinaire HAL, est destinée au dépôt et à la diffusion de documents scientifiques de niveau recherche, publiés ou non, émanant des établissements d'enseignement et de recherche français ou étrangers, des laboratoires publics ou privés. 


\title{
Recursive algorithms for the computation of the potential harmonic coefficients of a constant density polyhedron
}

\author{
Dimitrios Tsoulis ${ }^{1}$, Olivier Jamet ${ }^{2}$, Jérôme Verdun ${ }^{2,3}$, Nicolas Gonindard ${ }^{2,3}$ \\ ${ }^{1}$ Department of Geodesy and Surveying \\ Aristotle University of Thessaloniki \\ 54124 Thessaloniki, Greece \\ email: tsoulis@topo.auth.gr \\ ${ }^{2}$ Laboratoire de Recherche en Géodésie, IGN \\ 2 av. Pasteur, 94160 Saint-Mandé, France \\ email: Olivier.Jamet@ign.fr \\ ${ }^{3}$ Ecole Nationale des Sciences Géographiques, ENSG \\ 6 et 8 av. Blaise Pascal, Cité Descartes \\ 77455 Marne-La-Vallée CEDEX, France
}

Manuscript submitted to

Journal of Geodesy

2nd revised manuscript version of November 11, 2008 


\begin{abstract}
The gravitational potential of a constant density general polyhedron can be expressed both in terms of a closed analytical expression and as a series expansion involving the corresponding spherical harmonic coefficients. The latter can be obtained from two independent algorithms, which differ not only in their algorithmic architecture but in their efficiency and overall performance, especially when computing the coefficients of higher degree and order. In the present paper a comparative study of all these three approaches is carried out focusing on the numerical implementation of the recursive relations appearing in the two algorithms for the computation of the polyhedral potential harmonic coefficients. The performed numerical investigations show that the linear algorithm proposed by Jamet and Thomas (2004) but so far not implemented achieves a reasonable accuracy at a computational expense that opens to practical applications, for instance in the field of satellite gravimetry/gradiometry interpretation. The convergence behavior of the linear recursion algorithm is studied thoroughly and a computational procedure is proposed that enables the stable computation of potential harmonic coefficients up to degree 60 when referring to an arbitrarily shaped polyhedral body.
\end{abstract}

Keywords: Gravitational potential, general polyhedron, spherical harmonics, analytical expression, recursive algorithms 


\section{Introduction}

The use of the general polyhedron can be found in a wide range of applications in geodesy, geophysics and planetary sciences. Its unique geometry, i.e., its construction from an irregular number of faces, each built from a varying number of segments, defines an efficient and flexible modeling tool for the computation among others - of accurate terrain effects (Tsoulis 2001), or the representation of entire planetary bodies and the evaluation of the corresponding gravity signal (Simonelli et al 1993, Werner and Scheeres 1996).

For the practical evaluation of the polyhedral gravity signal there exist, in principle, three computational strategies: the direct numerical computation of closed analytical expressions which are available for the potential, its derivatives and the full gravity tensor for the case of the general polyhedron of constant density, the evaluation of the potential harmonic coefficients for the gravitational potential of the same body and numerical quadrature methods that can be applied directly to the corresponding integral expressions. The latter possibility is not going to be considered here.

The analytical formulas are valid everywhere in space including the polyhedron's faces, edges or vertices, where certain non zero singularity terms have to be taken into account (Petrović 1996, Tsoulis and Petrovic 2001). The fundamental property that is exploited for the derivation of the respective equations is the divergence theorem of Gauss, which permits the stepwise transformation of the initial three dimensional integral to a summation of a number of line integrals defined for the same number of segments building each face and leads to the evaluation of transcendental expressions involving the relative position of the coordinates of each vertex with respect to the computation point. Although the repeated application of the divergence theorem (for the transition from volume to surface and from surface to line integrals respectively) produces the aforementioned results in a straightforward manner, this is not the only way to proceed. Equivalent procedures have been proposed in the literature that are also valid everywhere but use different parametrization for the problem, for example the definition of the so-called solid angle subtended by a face when viewed from the field point (Werner 1994; Werner and Scheeres 1996). Apart from that, the task of deriving an analytical expression for the polyhedral gravity field has been dealt with from different algorithmic point of views by a variety of authors, e.g., Pohanka (1988), Barnett (1976) and many other contributions going back to the mid-1800s.

The computation of the potential harmonic coefficients of the general constant density polyhedron, which is an alternative way of evaluating the polyhedral gravity signal, is based on the expansion of its gravitational potential into an infinite spherical harmonics series expansion. The potential expansion in solid spherical harmonics has been always the preferable means of modeling the gravity field of an observed body in satellite-related applications. Thus, the polyhedral modeling has been applied successfully in studies of the gravity field of planetary objects for which satellite orbit perturbations were available. Formulating the expression for the potential in solid spherical harmonics and applying different strategies 
for the integration over the surface of the observed body one can be led to the corresponding harmonic coefficients of the body's gravitational potential. Thus, the spherical harmonic representation of the polyhedral gravity signal has been applied up to now in modelling the gravity field of planetary bodies such as asteroids or planets as a synthetic counterpart of the corresponding satellite-range related models (Chao and Rubincam 1989; Martinec, Peck and Bursa 1989; Balmino 1994).

As far as the algorithmic procedure is concerned, two basic approaches have been proposed so far for the spherical harmonic coefficients of the gravitational potential of a constant density general polyhedron. The first approach proposes the use of the representation of the radius vector of the integrand appearing in the general expression for the potential of the polyhedral source in terms of a spherical harmonic expansion. After introducing this expression into the respective equations which define the coefficients of the potential, one may proceed to the computation of these coefficients either numerically (Chao and Rubincam 1989) or analytically (Martinec, Peck and Bursa 1989; Balmino 1994). The second approach derives recurrent relations for the computation of these coefficients. Werner (1997) elaborated the well known recurrent relations for the associated Legendre functions in the integral expressions defining the sought coefficients. The basic geometric feature of his proposed algorithm is the partitioning of the polyhedron into a collection of simplices of tetrahedral shape. Then, the recurrent relations which he has derived for the harmonic coefficients for point masses and have to be integrated over the domain of the extended body, are expressed analytically over each tetrahedron in terms of certain trinomial expressions and then summed for each tetrahedron to give the final results for the harmonic coefficients. The trinomial expressions that are used in these approach are provided by the solution to the problem of integrating Cartesian-coordinated polynomials on a polyhedral domain as proposed by Lien and Kajiya (1984).

Both of the aforementioned approaches are restricted, each for different reasons, to the computation of harmonic expansions that are limited only to lower degrees. Werner's method, for example, requires the expansion of the respective polynomial integrands, for which the number of the necessary operations behaves as the square of the number of the harmonic coefficients that are going to be computed. Another method for the computation of the polyhedral potential harmonic coefficients has been proposed by Jamet and Thomas (2004). This method is also based on the division of the polyhedral source into a number of distinct tetrahedra and leads also to a recurrent solution. As for Werner's approach, the needed computational effort behaves thus linearly with respect to the number of tetrahedral simplices that have to be defined. However, in this case the recursions concern the integrals that define the coefficients. This defines an algorithm of linear complexity with respect to the number of computed coefficients, a fact that permits the computation of higher degrees. This latter alorithm was never implemented and one of the scope of the present paper concerns its assessment.

The present work presents a detailed numerical survey of the aforementioned recursive algorithms (Werner 
1997 \& Jamet and Thomas 2004). Their numerical implementation revealed that even the linear algorithm of Jamet and Thomas (2004) presented certain numerical deficiencies, when computed for higher degrees. In order to overcome this problem the algorithm is expanded to its normalized counterpart, something that increases the computational stability considerably and permits the computation of coefficients up to degree 400 and higher. The numerical implementation of these algorithms enabled not only their quantification and their direct comparisons with the closed analytical solution which are presented in this paper, but also revealed or verified numerically certain theoretical handicaps that are apparently inherited with the linear algorithm of Jamet and Thomas (2004). These numerical instabilities are documented in detail both theoretically and numerically. Finally a computational procedure is proposed that deals with these problems and enables the stable computation of a polyhedral gravity field for higher degrees. Using the shape model of asteroid 433 EROS as a test body we managed to apply this technique successfully and obtained a reproducible gravity field up to degree 60 .

\section{The linear algorithm}

The fundamental formula for the present discussion is the expression for the gravitational potential of an extended body as an infinite series expansion in solid spherical harmonics

$$
V(r, \theta, \lambda)=\frac{G M}{r}\left\{1+\sum_{n=1}^{\infty}\left(\frac{a}{r}\right)^{n} \sum_{m=0}^{n} P_{n, m}(\cos \theta)\left[C_{n, m} \cos (m \lambda)+S_{n, m} \sin (m \lambda)\right]\right\}
$$

where $G$ is the gravitational constant, $M$ stands for the total mass of the body and $a$ denotes a reference distance, often chosen as the radius of the smallest sphere centered at the origin which circumscribes the extended body. $(r, \theta, \lambda)$ are the spherical coordinates of the field point (radius, colatitude and longitude respectively, cf. Figure 2a) with respect to the coordinate origin and $P_{n, m}$ are the Legendre polynomials (for $m=0$ ) or associated Legendre functions (for $m>0$ ) as defined by Heiskanen and Moritz (1967).

For the dimensionless coefficients $C_{n, m}$ and $S_{n, m}$ the following integral expression holds

$$
\left[\begin{array}{c}
C_{n, m} \\
S_{n, m}
\end{array}\right]=\frac{2-\delta_{0, m}}{M a^{n}} \frac{(n-m) !}{(n+m) !} \iiint_{Q \in U} \mathbf{h}_{n, m}(Q) d u(Q)
$$

where $d u(Q)$ is the volume element of the total volume $U$ at point $Q\left(r_{Q}, \theta_{Q}, \lambda_{Q}\right)$ and the vector $\mathbf{h}_{n, m}(Q)$ has been inserted to denote the product between the radial distance of point $Q$ with the associated 
Legendre functions according to

$$
\mathbf{h}_{n, m}(Q)=r_{Q}^{n} P_{n, m}\left(\cos \theta_{Q}\right)\left[\begin{array}{c}
\cos \left(m \lambda_{Q}\right) \\
\sin \left(m \lambda_{Q}\right)
\end{array}\right]
$$

Thus, $\mathbf{h}_{n, m}(Q)$ defines a 'vector function' whose elements are functions of the spherical coordinates of point $Q$ with respect to the coordinate origin. The computation of equation 2 , or equally the integration of function $\mathbf{h}_{n, m}(Q)$ over the volume of a known polyhedral source of constant density is the scope of the contributions of Werner (1997) and Jamet and Thomas (2004). The common tool for both approaches is the division of the total volume $U$ of the polyhedron into a collection of simplices (tetrahedra), each having one vertex at the origin and the opposite face taken from one of the polyhedral faces (Figure 2 (b)). With this geometrical decomposition the volume integral of equation 2 can be expressed as the algebraic sum of the integrals over each simplex of the body $U$, where an integral is counted as positive if the outer normal to the polyhedron lies outside the simplex and negative otherwise. Thus, the sign of the respective contributions to the final value of the coefficients $C_{n, m}$ and $S_{n, m}$ depends on the relative position of each polyhedral face with respect to the respective tetrahedron.

To explain this we may consider the example of a cube. Its division into 6 simplices (one for each of its faces) of volume $U_{j}$ leads according to the distinction made above to the expression of its volume as $U=$ $\sum \delta_{j} U_{j}$, with $\delta_{j}$ equal to +1 for cases $4-6$ and -1 for cases 1-3, giving thus $U=-U_{1}-U_{2}-U_{3}+U_{4}+U_{5}+U_{6}$ (see Figure 1). After calculating the coefficients of all simplices, we will get for the harmonic coefficients of the cube the corresponding expression

$$
\left[\begin{array}{c}
C_{n, m} \\
S_{n, m}
\end{array}\right]=\sum \delta_{j}\left[\begin{array}{c}
C_{n, m}(j) \\
S_{n, m}(j)
\end{array}\right]
$$

with $\delta_{j}$ obtaining the same values as above.

In the following we will consider that $U$ denotes the volume of a simplex (tetrahedric element). Any polyhedron is decomposed into a set of such simplices (tetrahedra). The four faces building each one of these simplices will be denoted by $\sigma_{k}$, with $k=0, \ldots, 3, \sigma_{o}$ being the face that belongs to the polyhedron surface and $\sigma_{k}$, with $k=1, \ldots, 3$, the faces which share the origin as common vertex. The three edges of face $\sigma_{o}$ will be noted as $\varepsilon_{k}, k=1, \ldots, 3$, where $\varepsilon_{k}$ is the common edge between face $\sigma_{o}$ and face $\sigma_{k}$. The numbering of the edges is performed in the standard counterclockwise fashion, according to which the normal vectors to the corresponding faces will point in the outgoing direction of the respective tetrahedron. The normal vector to each face $\sigma_{k}$ will be denoted by $\mathbf{n}_{k}$ and the direction unit vector of each edge $\varepsilon_{k}$ will be noted $\mathbf{t}_{k}$. Finally, the involved spherical coordinates are defined by the standard expressions 


$$
\begin{aligned}
& x=r \sin \theta \cos \lambda \\
& y=r \sin \theta \sin \lambda \\
& z=r \cos \theta
\end{aligned}
$$

while the unit vector bases of the local spherical and cartesian frames will be given by $\left(\mathbf{u}_{r}, \mathbf{u}_{\theta}, \mathbf{u}_{\lambda}\right)$ and $\left(\mathbf{u}_{x}, \mathbf{u}_{y}, \mathbf{u}_{z}\right)$ respectively. An overview of all involved geometrical quantities is given in Figure 2.

We may introduce, for the sake of simplicity, the following parameter for the representation of the three dimensional integral in the right hand side of equation 2

$$
\mathbf{H}_{n, m}=\iiint_{Q \in U} \mathbf{h}_{n, m}(Q) d u(Q)
$$

with boldface denoting again a vector function, the simple notation $H_{n, m}$ representing the three dimensional integral of either component $h_{n, m}$ of the vector $\mathbf{h}_{n, m}$.

Taking into account the equality

$$
\begin{aligned}
\nabla \cdot\left(\frac{1}{n+3} r h_{n, m} \mathbf{u}_{r}\right) & =\frac{1}{r^{2}} \frac{\partial}{\partial r}\left(\frac{r^{3} h_{n, m}}{n+3}\right)=\frac{1}{r^{2}} \frac{\partial}{\partial r}\left(\frac{r^{n+3}}{n+3} P_{n, m}(\cos \theta)\left[\begin{array}{c}
\cos (m \lambda) \\
\sin (m \lambda)
\end{array}\right]\right) \\
& =r^{n} P_{n, m}(\cos \theta)\left[\begin{array}{c}
\cos (m \lambda) \\
\sin (m \lambda)
\end{array}\right]=h_{n, m}
\end{aligned}
$$

and applying the divergence theorem of Gauss to equation 2 gives

$$
H_{n, m}=\iiint_{U} h_{n, m} d u=\frac{1}{n+3} \iint_{\sigma_{o}} r h_{n, m} \mathbf{u}_{r} \cdot \mathbf{n}_{o} d \sigma
$$

Equation 7 transforms now at a first instance the three dimensional integral at the right hand side of equation 2 to a sole surface integral, which expresses the flow of the vector field $r h_{n, m} \mathbf{u}_{\mathbf{r}}$ through the triangular face that belongs to the polyhedral surface $\sigma_{o}$ and defines the specific tetrahedron, with $\mathbf{u}_{r}$ expressing the unit vector along the radial coordinate of the local spherical coordinate system, and $h_{n, m}$ 
denoting either component of the 'vector function' $\mathbf{h}_{\mathbf{n}, \mathbf{m}}$. The derivation of equation 7 expresses also the fact that vector $\mathbf{u}_{r}$ is per definition orthogonal to all three normal vectors $\mathbf{n}_{k}, k=1, \ldots, 3$, of the three tetrahedral faces not belonging to the polyhedron. Since all three faces building each simplex share the origin as common vertex, the direction vector $\mathbf{u}_{r}$ will be always orthogonal to the normal vectors $\mathbf{n}_{k}$ of each face.

From the geometrical setup of Figure $2 \mathrm{~b}$ the following relation can also be obtained directly

$$
r(Q) \mathbf{u}_{r}(Q) \cdot \mathbf{n}_{o}=d_{o}, \forall Q \in \sigma_{o}
$$

where $d_{o}$ is the distance of the origin from the plane defined by face $\sigma_{o}$. This leads to the expression

$$
H_{n, m}=\frac{d_{o}}{n+3} \iint_{\sigma_{o}} h_{n, m} d \sigma
$$

A subsequent application of the Stokes theorem can reduce each of these surface integrals into a set of three line integrals, each defined along the perimeter of the triangular polyhedral face.

Using the identity

$$
\begin{aligned}
\nabla \times\left[\begin{array}{c}
y h_{n, m} \\
-x h_{n, m} \\
0
\end{array}\right] & =(n+m)\left[\begin{array}{c}
x h_{n-1, m} \\
y h_{n-1, m} \\
z h_{n-1, m}
\end{array}\right]-(n+2)\left[\begin{array}{c}
0 \\
0 \\
h_{n, m}
\end{array}\right] \\
& =(n+m) r h_{n-1, m} \mathbf{u}_{r}-(n+2) h_{n, m} \mathbf{u}_{z}
\end{aligned}
$$

permits the application of the Stokes theorem for every segment $\varepsilon_{k}$ of the face $\sigma_{o}$. With $\left[y h_{n, m}-x h_{n, m} 0\right]$ being the corresponding vector function the theorem of Stokes leads for face $\sigma_{o}$ to the expression

$$
\sum_{k=1}^{3} \int_{\varepsilon_{k}}\left[\begin{array}{c}
y h_{n, m} \\
-x h_{n, m} \\
0
\end{array}\right] \cdot \mathbf{t}_{k} d l=(n+m) \iint_{\sigma_{o}} r h_{n-1, m} \mathbf{u}_{r} \cdot \mathbf{n}_{o} d \sigma-(n+2) \iint_{\sigma_{o}} h_{n, m} \mathbf{u}_{z} \cdot \mathbf{n}_{o} d \sigma(12)
$$

with $\mathbf{u}_{z}$ denoting the unit vector in the direction of the third coordinate of the cartesian frame, $\mathbf{n}_{o}$ the unit vector perpendicular to face $\sigma_{o}, d_{o}$ the distance between the origin and the plane of the face $\sigma_{o}, \mathbf{t}_{k}$ 
the direction unit vector (tangent vector) of each edge $\varepsilon_{k}$ and $d l$ the infinitesimal line element denoting the integration along these edges. Finally, $x$ and $y$ simply describe the two coordinates of the local cartesian frame (see Figure 2a).

Using equations 8 and 10 equation 12 becomes

$$
\begin{aligned}
H_{n, m}= & \frac{d_{o}(n+m)}{(n+3)\left(\mathbf{u}_{z} \cdot \mathbf{n}_{o}\right)} H_{n-1, m} \\
& -\frac{d_{o}}{(n+2)(n+3)\left(\mathbf{u}_{z} \cdot \mathbf{n}_{o}\right)} \sum_{k=1}^{3} \int_{\varepsilon_{k}}\left[\begin{array}{c}
y h_{n, m} \\
-x h_{n, m} \\
0
\end{array}\right] \cdot \mathbf{t}_{k} d l
\end{aligned}
$$

Equation 13 demonstrates that it is possible to compute the three dimensional integrals appearing on the right hand side of equation 2 in terms of a recurrence over degree $n$. At each step of this recurrence scheme the evaluation of three line integrals along the edges of face $\sigma_{o}$ is necessary. Jamet and Thomas (2004) showed that it is possible to evaluate each of these remaining line integrals through a set of recurrence relations as well. This leads finally to a nested recurrence scheme which delivers the integrals $H_{n, m}$. In order to comprehend the link between these two recursions, the recursive schema which can be developed for the computation of the line integrals appearing in the right hand side of equation 13 is going to be presented briefly in the following.

If one defines the curvilinear abscissa $s$ along each edge as a new parameter, choosing as an origin for its computation the point $\mathbf{o}_{k}$ on $\varepsilon_{k}$ that minimizes the distance $d$ from the origin of the spherical coordinate frame then we obtain the geometrical definitions (see Figure 2b)

$$
\begin{aligned}
&\left(o_{x}, o_{y}, o_{z}\right) \equiv \mathbf{o}_{k}, \text { the cartesian coordinates of the origin of the edge } \\
& d \equiv \sqrt{o_{x}^{2}+o_{y}^{2}+o_{z}^{2}} \quad, \text { the distance from } \mathbf{o}_{k} \text { to the origin of the frame } \\
&\left(t_{x}, t_{y}, t_{z}\right) \equiv \mathbf{t}_{k} \quad, \text { the cartesian components of vector } \mathbf{t}_{k} \\
& s_{\text {min }}, s_{\text {max }} \quad, \text { the bounds of the integration domain along the edge }
\end{aligned}
$$

and furthermore

$$
x=o_{x}+t_{x} s
$$




$$
\begin{aligned}
& y=o_{y}+t_{y} s \\
& z=o_{z}+t_{z} s \\
& r=d^{2}+s^{2}
\end{aligned}
$$

Through these definitions the line integrals of the right hand side of equation 13 become

$$
\int_{\varepsilon}\left[\begin{array}{c}
y h_{n, m} \\
-x h_{n, m} \\
0
\end{array}\right] \cdot \mathbf{t} d l=\left(t_{x} o_{y}-t_{y} o_{x}\right) \int_{\varepsilon} h_{n, m} d l
$$

Let us now use the symbol $I_{n, m}$ to denote the integral on the left hand side of equation 18 and $\mathbf{I}_{n, m}$ for the corresponding $2 \times 1$ matrix, according to the notation used so far. It will hold

$$
\mathbf{I}_{n, m}=\int_{\varepsilon} \mathbf{h}_{n, m} d l
$$

Similarly we define two additional integrals in matrix form which enter the subsequent calculations, namely

$$
\mathbf{J}_{n, m}=\int_{\varepsilon} s \mathbf{h}_{n, m} d l
$$

and

$$
\mathbf{K}_{n, m}=\int_{\varepsilon} s^{2} \mathbf{h}_{n, m} d l
$$

The link to the recurrence computation of these integral forms is obtained from the stable recurrence relation of the associated Legendre polynomials (Press et al. 1992, p. 253)

$$
(n-m) P_{n, m}(\xi)=\xi(2 n-1) P_{n-1, m}(\xi)-(n+m-1) P_{n-2, m}(\xi)
$$

with $\xi=\cos \theta$. Multiplying first both sides of this equation by $r^{n}\left[\begin{array}{c}\cos m \lambda \\ \sin m \lambda\end{array}\right]$ and then integrating with respect to $\varepsilon$, we finally get, by incorporating also expressions 5,16 and 17 , following recurrence formula 


$$
\begin{aligned}
(n-m) \mathbf{I}_{n, m}= & (2 n-1) o_{z} \mathbf{I}_{n-1, m}-(n+m-1) d^{2} \mathbf{I}_{n-2, m} \\
& +(2 n-1) t_{z} \mathbf{J}_{n-1, m}-(n+m-1) \mathbf{K}_{n-2, m}
\end{aligned}
$$

In order to be able to compute the recurrence scheme of equation 23, which relates $\mathbf{I}_{n, m}$ with $\mathbf{J}_{n, m}$ and $\mathbf{K}_{n, m}$, we need additional information. Indeed, if we calculate the first derivative of $\mathbf{h}_{n, m}$ with respect to $s$ we get

$$
\begin{aligned}
\left(d^{2}+s^{2}-\left(o_{z}+t_{z} s\right)^{2}\right) \frac{\partial}{\partial s} \mathbf{h}_{n, m} & =(n+m)\left(t_{z} d^{2}-o_{z} s\right) \mathbf{h}_{n-1, m} \\
& =-n\left(t_{z} o_{z}-\left(1-t_{z}^{2}\right) s\right) \mathbf{h}_{n, m} \\
& =+m\left(t_{x} o_{y}-t_{y} o_{x}\right)\left[\begin{array}{rr}
0 & 1 \\
-1 & 0
\end{array}\right] \mathbf{h}_{n, m}
\end{aligned}
$$

Integrating both sides of this equation for $\varepsilon$ gives

$$
\begin{aligned}
(n+2)\left(1-t_{z}^{2}\right) \mathbf{J}_{n, m}= & \left|\left(d^{2}+s^{2}-\left(o_{z}+t_{z} s\right)^{2}\right) \mathbf{h}_{n, m}\right|_{s_{\min }}^{s_{\max }} \\
& +(n+2) t_{z} o_{z} \mathbf{I}_{n, m}-m\left(t_{x} o_{y}-t_{y} o_{x}\right)\left[\begin{array}{rr}
0 & 1 \\
-1 & 0
\end{array}\right] \mathbf{I}_{n, m} \\
& -(n+m) t_{z} d^{2} \mathbf{I}_{n-1, m}+(n+m) o_{z} \mathbf{J}_{n-1, m}
\end{aligned}
$$

Following the same approach for the derivative of $s \mathbf{h}_{n, m}$ with respect to $s$ leads to the additional recursive relation

$$
\begin{aligned}
(n+3)\left(1-t_{z}^{2}\right) \mathbf{K}_{n, m}= & \left.\left|\left(d^{2}+s^{2}-\left(o_{z}+t_{z} \cdot s\right)^{2}\right) s \mathbf{h}_{n, m}\right|\right|_{s_{\min }} ^{s_{\max }} \\
& -\left(d^{2}-o_{z}^{2}\right) \mathbf{I}_{n, m}+(n+4) t_{z} o_{z} \mathbf{J}_{n, m} \\
& -m\left(t_{x} o_{y}-t_{y} o_{x}\right)\left[\begin{array}{rr}
0 & 1 \\
-1 & 0
\end{array}\right] \mathbf{J}_{n, m}
\end{aligned}
$$




$$
-(n+m) t_{z} d^{2} \mathbf{J}_{n-1, m}+(n+m) o_{z} \mathbf{K}_{n-1, m}
$$

Equations 23, 25 and 26 offer the complete recursion scheme for the computation of the line integrals in equation 13 through quantities $\mathbf{I}, \mathbf{J}$ and $\mathbf{K}$. The two major comments regarding these relations are (a) the strong dependencies between the three integrals, which leads to a nested computation of all three formulas and (b) the recurrent relationships apply to the degree $n$, with the order $m$ remaining constant. One consequence of (a) is of computational nature. When a general polyhedron is considered, part of the calculations giving the contributions of each resulting tetrahedron can be performed independently, which can facilitate a parallel computation. The consequence of (b) has an impact on the initializations of the recursive algorithm. Since $0 \leq m \leq n$, the computation has to be initialized with the values $\mathbf{I}_{n, n}$, $\mathbf{J}_{n, n}, \mathbf{K}_{n, n}$ and $\mathbf{H}_{n, n}$ for every $n$ smaller than the maximum degree that is to be computed. Taking $m=n$ and setting all terms for which $m>n$ equal to zero, we verify that equations 13,25 and 26 are valid. The same holds for equation 23 for $m=n-1$.

For the initialization of $\mathbf{I}_{n, n}$ we can use the identity

$$
P_{n, n}(\xi)=(2 n-1) \sqrt{1-\xi^{2}} P_{n-1, n-1}(\xi)
$$

to derive a relation between $\mathbf{h}_{n, n}$ and $\mathbf{h}_{n-1, n-1}$ according to

$$
\begin{aligned}
\mathbf{h}_{n, n} & =r^{n} P_{n, n}(\xi)\left[\begin{array}{c}
\cos n \lambda \\
\sin n \lambda
\end{array}\right] \\
& =(2 n-1) \sin \theta r^{n-1} P_{n-1, n-1}(\xi) r\left[\begin{array}{rr}
\cos \lambda & -\sin \lambda \\
\sin \lambda & \cos \lambda
\end{array}\right]\left[\begin{array}{l}
\cos n(\lambda-1) \\
\sin n(\lambda-1)
\end{array}\right] \\
& =(2 n-1)\left[\begin{array}{rr}
x & -y \\
y & x
\end{array}\right] \mathbf{h}_{n-1, n-1}
\end{aligned}
$$

Recalling the coordinate definition given by equation 5 and integrating equation 28 along $\varepsilon$ we get the sought iteration for $\mathbf{I}_{n, n}$

$$
\mathbf{I}_{n, n}=\left[\begin{array}{rr}
o_{x} & -o_{y} \\
o_{y} & o_{x}
\end{array}\right] \mathbf{I}_{n-1, n-1}+\left[\begin{array}{rr}
t_{x} & -t_{y} \\
t_{y} & t_{x}
\end{array}\right] \mathbf{J}_{n-1, n-1}
$$


where $\mathbf{J}_{n-1, n-1}$ can be obtained directly from equation 25 using only $\mathbf{I}_{n-1, n-1}$, with terms $\mathbf{I}_{n-2, n-1}$ and $\mathbf{J}_{n-2, n-1}$ being null.

By initializing the diagonal and subdiagonal terms in the recursions for the integrals $\mathbf{I}, \mathbf{J}$ and $\mathbf{K}$ one can compute all the other terms recursively for degree $n$ for a certain order $m$. Each step of these recurrences only requires the evaluation of the function $\mathbf{h}$ at the vertices of the tetrahedron. With all $I_{n, m}$ finally available one can calculate the volume integrals $H_{n, m}$ by the recurrence relationship 13 and deduce the values for the harmonic coefficients $C_{n, m}$ and $S_{n, m}$.

\section{Expansion of the linear algorithm to the normalized case}

If one increases $n$ and $m$ considerably then the $P_{n, m}$ obtain extremely large values and the coefficients $C_{n, m}$ and $S_{n, m}$ become very small. To avoid these numerical problems and permit the computation of high degree and order terms one can introduce the normalization factor (Heiskanen and Moritz 1967, eq. $(1-73))$

$$
N_{n, m}=\sqrt{\left(2-\delta_{0, m}\right)(2 n+1) \frac{(n-m) !}{(n+m) !}}
$$

with

$$
\delta_{0, m}=\left\{\begin{array}{cc}
1, & m=0 \\
0, & m \neq 0
\end{array}\right.
$$

The normalized coefficients and the normalized associated Legendre functions can then be computed according to

$$
\left[\begin{array}{c}
\overline{C_{n, m}} \\
\overline{S_{n, m}}
\end{array}\right]=\left[\begin{array}{c}
C_{n, m} / N_{n, m} \\
S_{n, m} / N_{n, m}
\end{array}\right]
$$

and

$$
\overline{P_{n, m}}=P_{n, m} N_{n, m}
$$

respectively. The inclusion of the normalization factor produces new recurrent relations for the linear algorithm presented in the previous section and these relations are going to be presented in the present section. 
Definitions. From equations 2 and 31, the values of normalized harmonic coefficient of the contribution of volume $U$ to the potential obey the following relation

$$
\left[\begin{array}{c}
\overline{C_{n, m}} \\
\overline{S_{n, m}}
\end{array}\right]=\frac{1}{N_{n, m}} \frac{2-\delta_{0, m}}{M a^{n}} \frac{(n-m) !}{(n+m) !} \iiint_{Q \in U} \mathbf{h}_{n, m}(Q) d u(Q)
$$

In order to simplify further the calculations, let us define the normalization factor $F_{n, m}$ as

$$
\begin{aligned}
F_{n, m} & \equiv \frac{1}{N_{n, m}} \frac{2-\delta_{0, m}}{M a^{n}} \frac{(n-m) !}{(n+m) !} \\
& =\frac{1}{M a^{n}} \sqrt{\frac{\left(2-\delta_{0, m}\right)(n-m) !}{(2 n+1)(n+m) !}}
\end{aligned}
$$

as well as the subsequent normalized quantities, in accordance with the definitions of the previous section

$$
\begin{aligned}
\overline{\mathbf{h}_{n, m}} & \equiv F_{n, m} \mathbf{h}_{n, m} \\
\overline{\mathbf{H}_{n, m}} & \equiv F_{n, m} \mathbf{H}_{n, m} \\
\overline{\mathbf{I}_{n, m}} & \equiv F_{n, m} \mathbf{I}_{n, m} \\
\overline{\mathbf{J}_{n, m}} & \equiv \frac{F_{n, m}}{a} \mathbf{J}_{n, m} \\
\overline{\mathbf{K}_{n, m}} & \equiv \frac{F_{n, m}}{a^{2}} \mathbf{K}_{n, m}
\end{aligned}
$$

As well as previously, non bold face letters will represent either component of the corresponding 'vector function'.

We will also need the following ratios

$$
\begin{aligned}
& R_{n, n-1}^{m} \equiv \frac{F_{n, m}}{F_{n-1, m}}=\frac{1}{a} \sqrt{\frac{(2 n-1)(n-m)}{(2 n+1)(n+m)}} \\
& R_{n, n-2}^{m} \equiv \frac{F_{n, m}}{F_{n-2, m}}=\frac{1}{a^{2}} \sqrt{\frac{(2 n-3)(n-m)(n-m-1)}{(2 n+1)(n+m)(n+m-1)}}
\end{aligned}
$$

Normalized recurrent relationships. With the previous definitions, the harmonic coefficients of the contribution of one simplex are

$$
\left[\begin{array}{c}
\overline{C_{n, m}} \\
\overline{S_{n, m}}
\end{array}\right]=\iiint_{Q \in \text { simplex }} \overline{\mathbf{h}_{n, m}}(Q) d u(Q)=\overline{\mathbf{H}_{n, m}}
$$


The multiplication of equation 13 by factor $F_{n, m}$ yields

$$
\begin{aligned}
\overline{H_{n, m}}= & \frac{R_{n, n-1}^{m} d_{o}(n+m)}{(n+3)\left(\mathbf{u}_{z} \cdot \mathbf{n}_{o}\right)} \overline{H_{n-1, m}} \\
& -\frac{d_{o}}{(n+2)(n+3)\left(\mathbf{u}_{z} \cdot \mathbf{n}_{o}\right)} \sum_{k=1}^{3} \int_{\varepsilon_{k}}\left[\begin{array}{c}
y \overline{h_{n, m}} \\
-x \overline{h_{n, m}} \\
0
\end{array}\right] \cdot \mathbf{t}_{k} d l
\end{aligned}
$$

where

$$
\begin{aligned}
\int_{\varepsilon_{k}}\left[\begin{array}{c}
y \overline{h_{n, m}} \\
-x \overline{h_{n, m}} \\
0
\end{array}\right] \cdot \mathbf{t}_{k} d l=\left(o_{y} t_{x}-o_{x} t_{y}\right) \int_{\varepsilon_{k}} \overline{h_{n, m}}(s) d s \\
=\left(o_{y} t_{x}-o_{x} t_{y}\right) \overline{I_{n, m}}
\end{aligned}
$$

The derivation of recurrent relationships between the $\overline{\mathbf{I}_{n, m}}, \overline{\mathbf{J}_{n, m}}$ and $\overline{\mathbf{K}_{n, m}}$ proceeds the same way, as for equations 23, 25 and 26. We obtain

$$
\begin{aligned}
(n-m) \overline{\mathbf{I}_{n, m}}= & (2 n-1) R_{n, n-1}^{m} o_{z} \overline{\mathbf{I}_{n-1, m}} \\
& -(n+m-1) R_{n, n-2}^{m} d^{2} \overline{\mathbf{I}_{n-2, m}} \\
& +(2 n-1) a R_{n, n-1}^{m} t_{z} \overline{\mathbf{J}_{n-1, m}} \\
& -(n+m-1) a^{2} R_{n, n-2}^{m} \overline{\mathbf{K}_{n-2, m}}
\end{aligned}
$$

and similarly

$$
\begin{aligned}
(n+2)\left(1-t_{z}^{2}\right) \overline{\mathbf{J}_{n, m}}= & \left|\frac{1}{a}\left(d^{2}+s^{2}-\left(o_{z}+t_{z} s\right)^{2}\right) \overline{\mathbf{h}_{n, m}}\right|_{s_{\text {min }}}^{s_{\max }} \\
& +(n+2) \frac{t_{z} o_{z}}{a} \overline{\mathbf{I}_{n, m}} \\
& -m \frac{\left(t_{x} o_{y}-t_{y} o_{x}\right)}{a}\left[\begin{array}{cc}
0 & 1 \\
-1 & 0
\end{array}\right] \overline{\mathbf{I}_{n, m}} \\
& -(n+m) R_{n, n-1}^{m} \frac{t_{z} d^{2}}{a} \overline{\mathbf{I}_{n-1, m}} \\
& +(n+m) R_{n, n-1}^{m} o_{z} \overline{\mathbf{J}_{n-1, m}}
\end{aligned}
$$

and

$$
(n+3)\left(1-t_{z}^{2}\right) \overline{\mathbf{K}_{n, m}}=\left|\frac{1}{a^{2}}\left(d^{2}+s^{2}-\left(o_{z}+t_{z} s\right)^{2}\right) s \overline{\mathbf{h}_{n, m}}\right|_{s_{\min }}^{s_{\max }}
$$




$$
\begin{aligned}
& -\frac{d^{2}-o_{z}^{2}}{a^{2}} \overline{\mathbf{I}_{n, m}}+(n+4) \frac{t_{z} o_{z}}{a} \overline{\mathbf{J}_{n, m}} \\
& -m \frac{\left(t_{x} o_{y}-t_{y} o_{x}\right)}{a}\left[\begin{array}{rr}
0 & 1 \\
-1 & 0
\end{array}\right] \overline{\mathbf{J}_{n, m}} \\
& -(n+m) R_{n, n-1}^{m} \frac{t_{z} d^{2} \overline{\mathbf{J}_{n-1, m}}}{a} \\
& +(n+m) R_{n, n-1}^{m} o_{z} \overline{\mathbf{K}_{n-1, m}}
\end{aligned}
$$

Equations 38, 40 and 41 require that $n>m$, while equation 39 applies for $n>m+1$. Though, as stated by Jamet and Thomas (2004), these recurrent relationships do extend to every pair $(n, m)$ of degree and order with $n \geq m$, by setting equal to zero all terms for which the degree $n$ is smaller than the order $m$.

\section{Werner's method}

An alternative iteration algorithm for the computation of the spherical harmonic coefficients for the potential of a polyhedral mass with constant density has been proposed by Werner (1997). In his approach Werner treats at a first level the general expression of the potential in spherical harmonics incorporating the standard recurrent relations for the associated Legendre functions as well for their fully normalized integrals. The obtained recurrences correspond to the integrands of $\overline{C_{n, m}}$ and $\overline{S_{n, m}}$ defined for individual point masses. In order to expand this formulation to an extended polyhedral body Werner implemented the polynomial integration technique over a polyhedral domain, proposed by Lien and Kajiya (1984). For the algorithmic details of the method the reader is referred to Werner (1997). His algorithm has been implemented here numerically in order to perform an independent test of our own derivations. Our calculations showed that the central transformation taking place in this procedure, i.e. the polynomial change of variables of the polyhedron's vertices, expresses one of the main handicaps of Werner's method, which creates a practically unbearable computational effort when attempting to compute coefficients of increasing degree and order.

\section{Comparative tests for simple case studies}

\section{Background}

In order to assess the two recursive algorithms, the one proposed by Werner (1997) and the one presented here, we set up some simple case studies of simple constant density tetrahedra and compare the 
harmonic expansion to the direct computation of closed analytical formulas. While the direct comparison between the two approaches is straightforward, the assessment of their accuracy though the comparison of the numerical value obtained from the series expansion of the potential with analytical values is more problematic. Series expansions yield band limited approximations of the value of the potential at a given point that are not directly comparable to the analytical values. To cope with this issue, we performed several numerical investigations.

The fundamental property which underlines all our experiments is the behavior of the kernel $1 / r_{P}$, which defines the decay of the potential with increasing distance of the computation point from the attracting source. Furthermore, for the present case of the expression of the potential in a spherical harmonic expansion the numerical behavior of $V$ is also fundamentally affected by the ratio $\left(r_{Q} / r_{P}\right)^{n}$, where $r_{Q}$ and $r_{P}$ are respectively the distance from the origin of the frame of the points $Q$ of the attracting source and of the computation point $P$. As $r_{P}$ becomes larger the convergence rate for the series expansion increases. As $r_{P}$ becomes smaller the series convergence is not guaranteed. More precisely, the series converges for all $P$ s located outside a sphere enclosing all masses $\left(r_{Q}<r_{P}\right)$; for $r_{P}<r_{Q}$ the area of convergence is again a sphere, such that the mass distribution is nowhere included or intersected (Tsoulis 1999).

The above well known theoretical assets of spherical harmonic expansions led us to set up a first experiment aiming at assessing the convergence of the series expansion with respect to (a) increasing degree and order and (b) increasing values of $r_{P}$. The second experiment consists in studying the convergence of the series through the computation of high degree and order expansions, and will sketch an integrated view on the accuracy of the coefficients.

Both these experiments apply only to the normalized Jamet and Thomas's algorithms presented in this paper, as stated below. We will thus previously perform a direct comparison of both methods at low degrees.

Direct comparison

For the numerical implementation of the aforementioned tests a simple prismatic source as the one shown in Figure $2 \mathrm{~b}$ has been defined. It is a simple tetrahedron, since both existing recurrence algorithms for the evaluation of the potential harmonic coefficients (Werner's approach and the linear algorithm presented previously) refer to a tetrahedral geometry. Furthermore it is identical to the test body used by Werner (1997) for the numerical assessment of his own recursive algorithm. This choice has been made deliberately, in order to draw a first immediate conclusion on the validity of our approach. Following Werner (1997) the constant density of the tetrahedral mass has been also taken equal to $5.52 \mathrm{~g} / \mathrm{cm}^{3}$ and 
the origin of the local coordinate system has been defined as one of the four vertices. The two matrices needed for the implementation of the linear algorithm presented here are the coordinates of the four vertices and their linkage, which forms an extra topology matrix with the precondition that the order of the vertices will be such, that the normal vector to each of the corresponding planes will point outside the tetrahedron. Thus, the two matrices $G$ and $H$ read for our test tetrahedron correspondingly

$$
G=\left[\begin{array}{ccc}
-2 & -1 & 1 \\
1 & 0 & 1 \\
0 & 1 & 1 \\
0 & 0 & 0
\end{array}\right] \quad, \quad H=\left[\begin{array}{ccc}
1 & 2 & 3 \\
1 & 4 & 2 \\
3 & 4 & 1 \\
2 & 4 & 3
\end{array}\right]
$$

The obtained numerical values for the coefficients $C_{n, m}$ and $S_{n, m}$ up to degree 4 applying the linear recursive algorithm read

$$
\mathbf{C}=\left[\begin{array}{ccccc}
1.6727272727 & 0 & 0 & 0 & 0 \\
0.2851622661 & -0.0950540886 & 0 & 0 & 0 \\
0.0463802081 & -0.0401664385 & 0.0200832192 & 0 & 0 \\
0 & -0.0086628747 & 0.0124520069 & -0.0030501063 & 0 \\
-0.0033967950 & 0.0021180637 & 0.0042791349 & -0.0024016585 & -0.0002830382
\end{array}\right]
$$

and

$$
\mathbf{S}=\left[\begin{array}{ccccc}
0 & 0 & 0 & 0 & 0 \\
0 & 0 & 0 & 0 & 0 \\
0 & 0 & 0.0200832193 & 0 & 0 \\
0 & 0.0023626022 & 0.0124520069 & -0.0091503189 & 0 \\
0 & 0.0027232248 & 0.0040651782 & -0.0072049755 & 0.0039625344
\end{array}\right]
$$

where we have used a matrix notation, with rows expressing the degree $n$ and columns the order $m$. These values are identical with those given by Werner (1997, Table 1) down to the numerical level of 1e-16, which corresponds to the intrinsic precision of the personal computer.

Although both methods produce identical results, they perform differently in terms of computational efficiency. For degrees $n<10$ the required CPU time for both is comparable. However, when computing the higher degrees the differences become very significant. Taking the same tetrahedron and computing its potential harmonic coefficients for increasing degree and order using both methods we get a similar evolution of the required CPU time, which increases exponentially with respect to the increased degree. 
However, this exponential behavior refers to different order of magnitudes. Thus, the linear algorithm requires 16 seconds in order to produce the coefficients of the aforementioned tetrahedron up to degree and order 360, whereas the Werner method consumes about 350 seconds of CPU time for the computation of the coefficients only up to degree and order 50. The increase of CPU time behaves exponentially, almost approximating a function of degree four. Thus, the necessary computer time becomes very quickly unmanageable, leading approximately to $9.4 \mathrm{e} 5$ seconds (approximately 11 days) of required CPU time for the computation of the coefficients up to degree and order 360 of a single tetrahedron on the same standard contemporary PC. The introduction of the coordinate mapping through the corresponding polynomials which enables the final integration step in the Werner method, produces an enormous computational effort and makes the method inapplicable for higher degrees and more complicated bodies.

Hence, from both available recurrent algorithms the linear integration algorithm proves to be a much more efficient method, though producing the same level of accuracy as the method proposed by Werner. The linear algorithm is superior in terms of required CPU time for the lower degrees and is the only applicable method for the higher degrees. When applied to a complex body, for which a decomposition into a number of simple tetrahedra as many as the body's faces is necessary, the corresponding CPU time would increase linearly as to the total number of these faces. On the same computer the expansion up to degree 400 requires 22 seconds for a single tetrahedron, and would require approximately 100 days for the expansion up to the same degree of the potential of a body counting 400,000 faces - for instance a DTM at the resolution $25 \mathrm{~km}$ over the whole Earth. This still represents a heavy computation burden, but would become manageable if shared among a few tens of CPUs. Hence, our recursive approach may allow some applications at the scale of the whole Earth, such as, for instance, an exact removal of the effect of the topography from the gravity signal of the future GOCE satellite, which would enhance our interpretation capabilities.

Comparison to analytically derived values

In order to assess the precision of the derived recurrent algorithm one has to compare it with the corresponding analytical solution at a given point $P$. A detailed evaluation should investigate the main factors that define the recurrent relationships numerically, namely the maximum degree of the expansion and the position of the computation point with respect to the attracting source. As a general rule of thumb it holds that a higher degree of expansion into spherical harmonics produces potential values that are closer to the (exact) analytical ones. Furthermore, as stated before, the further the computation point gets with respect to the attracting source the smaller the numerical value of the ratio $\left(\frac{r_{q}}{r_{p}}\right)^{n}$ becomes, $Q$ symbolizing any point of the attracting source. This well known numerical asset of spherical harmonics implies that higher degree coefficients are more important to the precision of the corresponding potential values the closest one gets to the attracting source. On the contrary, one should expect that a small 
number of coefficients would suffice for reaching the desired agreement with the analytical solution when the computation point is located in a fairly large distance from the prismatic source.

To assess more precisely this effect, we compare the convergence with increasing $r_{P}$ of the series truncated at a given degree with the convergence of the series obtained for a point mass located at the center of mass of the studied tetrahedron. As any body with a certain spatial extent should generate a potential with more energy in the low frequencies than a point mass, we expect that the point mass convergence should be better for series truncated at a very low degree, and should get worse for series truncated some higher degree.

Table 1 presents the comparison of the tetrahedron truncated potential series expansions at points $P$ of increasing distance $r_{P}$ from the origin of the frame with analytical values. The corresponding exact numerical value for the potential of the source tetrahedron at every location of $P$ is evaluated through the closed analytical expressions for the general constant density polyhedron, which are valid everywhere in space including all possible singularity terms, as given by Tsoulis and Petrović (2001).

We use again the model body of Figure 2b, with equation 42 describing its geometry. The series expansion, expressed by equation (1) with $r_{p}$ playing the role of $r$, is actually an expansion in powers of $1 / r_{p}$. For increasing $r_{p}$ the convergence of the series becomes better, for smaller $r_{p}$ this convergence cannot be guaranteed. With the origin of the local coordinate system being situated at vertex 4 of the tetrahedron we studied the aforementioned expansion for degrees 2 and 10 using the coefficients obtained by the presented recursive linear algorithm. The computations took place at eleven distinct points, namely starting at $[-2-1-1]$ and gradually getting at point $[-4-2-2]$ with respect to the same coordinate system. Table 1 summarizes these results giving also the respective analytical values and the corresponding differences. The obvious remark taken from these values is that when the distance from the tetrahedron increases the expansion becomes more precise for the same degree, with the differences between these two specific degrees decreasing in value for the same point as this moves away from the tetrahedron. This observation proves the expected behavior of the harmonics $C_{n, m}$ and $S_{n, m}$ to obtain smaller numerical values both for increasing $n$ and for increasing distance $r_{p}$. Consequently, the closer the computation point $P$ and the attracting point $Q$ get, the higher is the difference between the contributions of the same degree, in other words one has to use a higher degree expansion to be as accurate.

The convergence with increasing $r_{P}$ of these series truncated at a given degree is compared to the convergence of identically truncated series expansion of the potential of a mass point located at the center of mass of the tetrahedron in Table 2. The spherical harmonic expansion of the potential of a mass point is derived after the well known expression (Heiskanen and Moritz, 1967)

$$
\frac{1}{P Q}=\frac{1}{r_{P}}\left(1+\sum_{n=1}^{+\infty}\left(\frac{r_{Q}}{r_{P}}\right)^{n} P_{n}(\cos \hat{P Q})\right)
$$


where $P Q$ is the distance between $P$ and $Q$, and where $\hat{P Q}$ is the angle between the directions of $P$ and $Q$ at the origin of the frame. Table 2 presents, for each value of $r_{P}$, the error of the truncated series at degrees 2 and 10 in percentage to the analytical value of the potential. As expected, we observe that, at degree 2 , the series of the tetrahedron converges more slowly with $r_{P}$ than the corresponding series of the point mass, while at degree 10, the series of the tetrahedron does converge more quickly. While this experiment does not allow to quantify the accuracy of the individual coefficients of the series, it shows that the implemented algorithm does behave as expected, allowing thus to assess the correctness of the implementation at low degrees.

Convergence at high degrees

Table 1 reveals a certain trend for the potential as the location of the computation point increases. In order to underline this trend and furthermore demonstrate the role of the distance between $P$ and the tetrahedron for the convergence of the corresponding series expansions we proceeded to the numerical tests summarized in Figures 3-5. The computations take place at two locations, namely [-4 -4 0] (Figure 3 ) and $\left[\begin{array}{ll}-2 & -2\end{array}\right]$ (Figures 4 and 5). In these graphs the relation between the expansion degree and the precision with respect to the analytical values becomes more evident. For an increased distance from the tetrahedron and a given degree the spherical harmonic expansion for the potential becomes more accurate. The series convergence is also affected by the relative position of $P$ with respect to the tetrahedron. The more distant point (Figure 3) causes a rapid convergence of the series which coincides with the analytical solution already by degree 6 at the 2e-13 level and from degree 7 upwards at the 1e-15 level. On the other hand, the nearest location of the computation point leads to a series expansion that converges only after degree 25. Up to that degree the series expansion for the potential oscillates around the analytical solution showing a much slower convergence as Figure 4 and the first part of Figure 5 demonstrate. The agreement between the series expansion and the analytical solution is 9e-14 for degree 25 and it oscillates around 1e-15 from degree 30 up to degree 50 (Figure 5, right part).

The magnitude of these differences, especially the oscillating behavior of the series expansion solution with respect to the analytical solution for increasing degree, intrigued us to explore their development for even higher degrees. Thus, we extended these comparisons for the same positions of $P$ incorporating coefficients $C_{n, m}$ and $S_{n, m}$ up to degree and order 360 . This led to a very interesting numerical finding. The levels of accuracy obtained for the two cases are namely not identical. The accuracy, or equally the level of agreement between series expansion and analytical solution, that is achieved for the 'near' situated point $\left(\left[\begin{array}{lll}-2 & -2 & 0\end{array}\right]\right)$ is less than the one obtained for the 'far' point $\left(\left[\begin{array}{lll}-4 & -4 & 0\end{array}\right]\right)$, the actual values being 1e-17 and 1e-22 respectively. Furthermore, these upper accuracy bounds are reached at different degrees. The 1e-22 accuracy level is obtained for the more distant point already by degree 30 , while the 1e-17 accuracy level is reached at the nearest point only after degree 100. This finding quantifies a bit 
more precisely the numerical accuracy of the recurrent scheme. The computation of the series expansion at the 'near' point yield an estimate of the accuracy of the series at degree and order 100, while the same computation at the 'far' point yields an estimate of the accuracy of the series at degree and order 30: the energy of the potential at higher degrees appears to be lesser than the accuracy of the algorithm at the given locations. As expected, the accuracy gets worse as the expansion is computed for higher degrees and orders. Though, the increase of the error keeps reasonable with respect to the intrinsic accuracy of the computer itself.

Divergence cases

All of the aforementioned results referred to a specific choice for the origin of the local coordinate system, namely one of the corners of the tetrahedron. In order to test the method in a more general case, namely to mimic the case of the computation of the spherical harmonic decomposition of the potential of a geological structure, we chose to keep our test tetrahedron and to move the origin of the frame relatively to it. For these computations the tetrahedron is handled as a four-faced prismatic source with the final value of the coefficients being obtained according to equation 4 . Thus, the source tetrahedron is divided each time into 4 simplices all of which have the origin as their common vertex and the computation proceeds according to Figure 1 and equation 4.

Our numerical investigations revealed that the choice of the origin is mostly critical for the overall stability of the process. In simple words, any displacement of the origin away from its initial location makes the method immediately divergent.

This fact is demonstrated briefly in Figure 6. The position of both the source tetrahedron and the computation point remain unaltered relative to each other. This implies that the corresponding potential values should be insensitive to the actual location of the origin of the local coordinate system. We then start to move gradually the origin from its original location (vertex 4 ) along the $x$-axis. As Figure 6 demonstrates, the deviation of the series expansion from the analytical value is abrupt. This instability characterizes all degrees, although Figure 6 deals with a maximum degree and order of 360 .

Thus, the linear recursive algorithm which permits an efficient and accurate computation of the spherical harmonic coefficients that describe the gravitational potential of a constant density polyhedron is inherited with severe numerical instabilities, that cause immediate divergence of the method, depending on the location of the origin of the local coordinate system. This instability has a solid theoretical explanation and is linked with the actual orientation of the tetrahedral faces with respect to the coordinate system, as will be demonstrated in the following section. 


\section{Method assessment}

The severe numerical instabilities that were outlined through the computations of the coefficients of the tetrahedron for various origins of the local coordinate system can be interpreted as a numerical assessment of patent theoretical weaknesses of the linear method. In this section, we show that the recurrent scheme initially proposed by Jamet and Thomas (2004) and normalized previously is inherently unstable.

Volume integrals

Assuming that the line integrals $\overline{\mathbf{I}_{\mathbf{n}, \mathbf{m}}}$ are properly estimated, the volume integrals $\overline{H_{n, m}}$ are derived from the scalar autoregressive relation 38. The stability of this computation is ensured if and only if the propagation factor $\alpha_{n}=R_{n, n-1}^{m} \frac{d_{0}(n+m)}{(n+3)\left(\mathbf{u}_{\mathbf{z}} \cdot \mathbf{n}_{\mathbf{0}}\right)}$ is smaller than 1 . This factor verifies the relation

$$
\alpha_{n}=\sqrt{\frac{(2 n-1)\left(n^{2}-m^{2}\right)}{(2 n+1)(n+3)^{2}}} \frac{d_{0}}{a} \frac{1}{\mathbf{u}_{z} \cdot \mathbf{n}_{o}}<\frac{d_{0}}{a} \frac{1}{\mathbf{u}_{z} \cdot \mathbf{n}_{o}}
$$

where $d_{0}$ is the distance between the origin of the frame and the plane of the considered face of the polyhedron, $\mathbf{u}_{z} \cdot \mathbf{n}_{o}$ the cosine of the angle between the normal to this face and the vertical direction and $a$ is the chosen reference distance.

For large degrees $n, \alpha_{n}$ approaches its upper limit as

$$
\lim _{n \rightarrow+\infty} \alpha_{n}=\frac{d_{0}}{a} \frac{1}{\mathbf{u}_{z} \cdot \mathbf{n}_{o}}
$$

The computation of large degrees is thus only possible for the faces of the considered polyhedron that verify

$$
\frac{d_{0}}{a} \frac{1}{\mathbf{u}_{z} \cdot \mathbf{n}_{o}}<1
$$

For a given tetrahedron with a horizontal upper face such as our test body, there always exists a rotation around the origin of the frame for which $\mathbf{u}_{\mathbf{z}} \cdot \mathbf{n}_{\mathbf{o}}$ becomes as small as wished, that is for which $\alpha_{n}>1$. In other words, the recurrent relationship linking the volume integrals does not allow the computation of the spherical harmonic coefficient for any given face geometry and orientation.

Moreover, the reference distance $a$ is generally chosen as small as possible, since $a$ is also the smallest distance $r$ to the origin for which the series of spherical harmonic representing the potential (equation 1 ) converges. In practical applications, $a$ defines the smallest sphere enclosing all masses of the considered 
body. The ratio $\frac{d_{0}}{a}$ is thus very likely to be very close to 1 for at least one face of the considered polyhedron. Consequently the recurrent scheme will only apply when theses faces are horizontal in the chosen reference frame.

For theses reasons, the proposed algorithm will only apply to restricted cases of tetrahedra (the reference frame beeing given). In particular, it can be applied to any tetrahedron whose face opposite to the origin is horizontal.

Line integrals

The computation of the line integrals makes use of more complex recurrent relationships. Let $\mathbf{V}_{\mathbf{n}, \mathbf{m}}$ be the 6 component vector defined as

$$
\mathbf{V}_{\mathbf{n}, \mathbf{m}}=\left[\begin{array}{l}
\overline{\mathbf{I}_{\mathbf{n}, \mathbf{m}}} \\
\overline{\mathbf{J}_{\mathbf{n}, \mathbf{m}}} \\
\overline{\mathbf{K}_{\mathbf{n}, \mathbf{m}}}
\end{array}\right]
$$

where $\overline{\mathbf{I}_{\mathbf{n}, \mathbf{m}}}, \overline{\mathbf{J}_{\mathbf{n}, \mathbf{m}}}$ and $\overline{\mathbf{K}_{\mathbf{n}, \mathbf{m}}}$ are the 2 component vectors corresponding to the line integrals defined by equation 35 . The recurrent relationships 39,40 and 41 can then be written in matrix form as follows

$$
\mathbf{V}_{\mathbf{n}, \mathbf{m}}=M_{0} \mathbf{V}_{\mathbf{n}, \mathbf{m}}+M_{1} \mathbf{V}_{\mathbf{n}-\mathbf{1}, \mathbf{m}} M_{2} \mathbf{V}_{\mathbf{n}-\mathbf{2}, \mathbf{m}}+\mathbf{D}_{\mathbf{n}}
$$

where $M_{0}, M_{1}$ and $M_{2}$ are three $6 \times 6$ matrices defined as

$$
\begin{aligned}
& M_{0}=\left[\begin{array}{cccccc}
0 & 0 & 0 & 0 & 0 & 0 \\
0 & 0 & 0 & 0 & 0 & 0 \\
\frac{t_{z} o_{z}}{a\left(1-t_{z}^{2}\right)} & -\frac{m}{n+2} \frac{\left(t_{x} o_{y}-t_{y} o_{x}\right)}{a\left(1-t_{z}^{2}\right)} & 0 & 0 & 0 & 0 \\
\frac{m}{n+2} \frac{\left(\frac{\left.t_{x} o_{y}-y_{y} o_{x}\right)}{a\left(1-z_{z}^{2}\right)}\right.}{\frac{t_{z}}{a\left(1-t_{z}^{2}\right)}} & 0 & 0 & 0 & 0 & 0 \\
-\frac{1}{n+3} \frac{d^{2}-o_{z}}{a^{2}\left(1-t_{z}^{2}\right)} & 0 & \frac{n+4}{n+3} \frac{t_{z} o_{z}}{a\left(1-t_{z}^{2}\right)} & -\frac{m}{n+3} \frac{\left(t_{x} o_{y}-t_{y} o_{x}\right)}{a\left(1-t_{z}^{2}\right)} & 0 & 0 \\
0 & -\frac{1}{n+3} \frac{d^{2}-o_{z}^{2}}{a^{2}\left(1-t_{z}^{2}\right)} & \frac{m}{n+3} \frac{\left(t_{x} o_{y}-t_{y} o_{x}\right)}{a\left(1-t_{z}^{2}\right)} & \frac{n+4}{n+3} \frac{t_{z} o_{z}}{a\left(1-t_{z}^{2}\right)} & 0 & 0
\end{array}\right] \\
& M_{1}=R_{n, n-1}^{m}\left[\begin{array}{cccccc}
\frac{2 n-1}{n-m} o_{z} & 0 & \frac{2 n-1}{n-m} a t_{z} & 0 & 0 & 0 \\
0 & \frac{2 n-1}{n-m} o_{z} & 0 & \frac{2 n-1}{n-m} a t_{z} & 0 & 0 \\
-\frac{n+m}{n+2} \frac{t_{z} d^{2}}{a\left(1-t_{z}^{2}\right)} & 0 & \frac{n+m}{n+2} \frac{o_{z}}{1-t_{z}^{2}} & 0 & 0 & 0 \\
0 & -\frac{n+m}{n+2} \frac{t_{z} d^{2}}{a\left(1-t_{z}^{2}\right)} & 0 & \frac{n+m}{n+2} \frac{o_{z}}{1-t_{z}^{2}} & 0 & 0 \\
0 & 0 & -\frac{n+m}{n+3} \frac{t_{z} d^{2}}{a\left(1-t_{z}^{2}\right)} & 0 & \frac{n+m}{n+3} \frac{o_{z}}{1-t_{z}^{2}} & 0 \\
0 & 0 & 0 & -\frac{n+m}{n+3} \frac{t_{z} d^{2}}{a\left(1-t_{z}^{2}\right)} & 0 & \frac{n+m}{n+3} \frac{o_{z}}{1-t_{z}^{2}}
\end{array}\right](49)
\end{aligned}
$$




$$
M_{2}=-\frac{n+m+1}{n-m} R_{n, n-2}^{m}\left[\begin{array}{cccccc}
d^{2} & 0 & 0 & 0 & a^{2} & 0 \\
0 & d^{2} & 0 & 0 & 0 & a^{2} \\
0 & 0 & 0 & 0 & 0 & 0 \\
0 & 0 & 0 & 0 & 0 & 0 \\
0 & 0 & 0 & 0 & 0 & 0 \\
0 & 0 & 0 & 0 & 0 & 0
\end{array}\right]
$$

and where $\mathbf{D}_{\mathbf{n}, \mathbf{m}}$ is a 6 component vector of inputs at each step of the recurrence.

The 6 component vectorial second order recurrent relationship of equation 47 can be expressed as a 12 component vectorial recurrent relationship of order 1 as follow

$$
\begin{aligned}
{\left[\begin{array}{c}
\mathbf{V}_{\mathbf{n}, \mathbf{m}} \\
\mathbf{V}_{\mathbf{n}-\mathbf{1}, \mathbf{m}}
\end{array}\right] } & =\left[\begin{array}{cc}
\left(1-M_{0}\right)^{-1} M_{1} & \left(1-M_{0}\right)^{-1} M_{2} \\
1 & 0
\end{array}\right]\left[\begin{array}{c}
\mathbf{V}_{\mathbf{n}-\mathbf{1}, \mathbf{m}} \\
\mathbf{V}_{\mathbf{n}-\mathbf{2}, \mathbf{m}}
\end{array}\right]+\left[\begin{array}{c}
\mathbf{D}_{\mathbf{n}} \\
0
\end{array}\right] \\
& \equiv A\left[\begin{array}{c}
\mathbf{V}_{\mathbf{n}-\mathbf{1}, \mathbf{m}} \\
\mathbf{V}_{\mathbf{n}-\mathbf{2}, \mathbf{m}}
\end{array}\right]+\left[\begin{array}{c}
\mathbf{D}_{\mathbf{n}} \\
0
\end{array}\right]
\end{aligned}
$$

The stability of this recurrent scheme depends on the eigenvalues of the matrix $A$ as defined by equation 51. Theses eigenvalues do not have a simple analytical expression, and we cannot derive a general theory about the divergences of the recurrent computations. Considering that the computation of the volume integrals applies mainly to horizontal faces, and that the method should apply to faces lying close to the sphere of radius $a$, we focus on the situation when

$$
\begin{aligned}
t_{z} & =0(\text { horizontal case }) \\
d & \approx a(\text { edge close to the tangent to the convergence sphere })
\end{aligned}
$$

and investigate the behavior of the eigenvalues of matrix $A$ for large degree and order.

Setting the ratio $\frac{n}{m}$ as a constant, one can show that matrix $A_{\infty} \equiv \lim _{m \rightarrow+\infty} A$ depends on 3 parameters :

$$
\begin{aligned}
\text { the ratio } & d_{a} \equiv \frac{d}{a} \in[0,1] \\
\text { the ratio } & k \equiv \frac{n}{m} \in[1,+\infty[ \\
\text { a position factor } & f \equiv \frac{\sqrt{d^{2}-o_{z}^{2}}}{a} \in[0,1]
\end{aligned}
$$


The ratio $d_{a}$ indicates whether the line carrying the considered edge of the body is close (small ratio) to the origin of the frame or not (high ratio). The ratio $k$ increases though the recurrent steps of the computation ( $k=1$ at initialization). The position factor $f$ is linked with the position of the line carrying the edge; $o_{z}$ is the $z$ coordinate of the closest point of this line to the origin of the frame. $f$ is small when the edge is close to the poles of the convergence sphere of radius $a$ and it increases as the edge comes closer to the equator.

Figure 7 plots the maximum eigenvalue of matrix $A_{\infty}$, for $d_{a}=1$, as a function of $k$ and $f$. It shows that for small values of $k$, that is for the first steps of the recurrence, the recurrence is always divergent (with a maximum eigenvalue greater than 1), whatever the position of the edge might be. Figure 7 also shows that the recurrence relationships tend to become stable as the ratio $k=\frac{n}{m}$ increases. This test computation of the eigenvalues of the recurrence matrix illustrates the behavior of the process for very large values of the computed order, and for edges nearly tangential to the convergence sphere of radius $a$.

For the test tetrahedron presented in the previous section, we ran a computation of the spherical harmonic coefficients with a random perturbation of the last significant digit of the input values of equations 40 (computation of $\overline{\mathbf{J}_{\mathbf{n}, \mathbf{m}}}$ ) and 41 (computation of $\overline{\mathbf{K}_{\mathbf{n}, \mathbf{m}}}$ ). The comparison between the values issued from this perturbated computation and the values obtained without perturbation yields a numerical estimate of the stability of the line integral recurrence procedure (the computation of the volume integral for a horizontal face being stable). Figure 8 presents an estimate of the relative accuracy of the coefficients obtained this way (ratio of difference between perturbated and not perturbated results and the result itself). The two diagonals (dashed lines) correspond to the coefficients $\overline{C_{n, n}}$ and $\overline{S_{n, n}}$ (initialization of the recurrence), and the recurrent computation is processed along the verticals of the drawing. One can notice that the computation errors increase at the beginning of the recurrence, for low values of the ratio $\frac{n}{m}$, and then decrease as the degree $n$ increases for any given order $m$. This observation is in complete agreement with the behavior of the eigenvalues of the matrix $A_{\infty}$ shown in Figure 7.

\section{Application}

We conclude from the previous analysis that, while the volume recurrent computation can be made stable by choosing a proper reference frame in which the face of the tetrahedron opposite to the origin is horizontal, the line recurrent computations are essentially divergent. Nevertheless, considering that i) the proposed algorithm is linear and thus computationally much more efficient that other existing approaches, and ii) that the perturbation method presented in the previous section seems to allow the computation of a reasonable estimate of the accuracy loss due to this instability, we propose in this section a computational strategy for deriving the spherical harmonic coefficients of the potential of a constant 
density polyhedron based on the present recurrent relationships, and assess the results obtained for a test body.

\section{Computational strategy}

The approach we implemented proceeds as follows. The considered polyhedral body is triangulated and divided into tetrahedra having each one vertex at the origin of the reference frame, and a face coinciding with one triangular face of the body.

The spherical harmonic coefficients of the potential of each tetrahedron are computed up to a given degree and order $N$ in a local frame rotated around the origin of the reference frame in such a way that the considered face of the body will be horizontal. This computation is performed twice, once without perturbation, and once with a perturbation of the last significant digit of the input values of the line integrals recurrent relationships. All the coefficients for which the two estimates differ by more that $10^{-2}$ of the estimated values are set to zero.

The harmonic coefficients of the potential of the whole body in the initial reference frame are then obtained by cumulating the individual face contributions after rotating the spherical harmonic decomposition of each face from its own frame to the initial reference frame. In order to avoid the computation of a spherical harmonic rotation matrix for each face of the body, this rotation is achieved through the computation, for each degree $n \leq N$, of the total potential of the whole body at $3(2 n+1)$ points homogeneously distributed on a sphere of radius $a$. This degree $n$ potential is then inverted into a spherical harmonic decomposition at degree $n$ (on the basis of the $2 n+1$ spherical harmonic functions of order $m \leq n$ ).

Test body

The results presented here were computed for a triangulated shape model of the asteroid 433 EROS (approximate size $13 \times 13 \times 33 \mathrm{~km}$ ), which was mapped by the NEAR Shoemaker probe from April to October 2000. Several models of this body at various resolutions have been made available through the internet by the Planetary Science Institute (USA). We used the model 'eros001708' consisting of 1708 faces, presented in Figure 9 and available at http://www.psi.edu/pds/archive/shape.html.

Results

The spherical harmonic coefficients of the used shape model of 433 EROS were computed with a reference distance $a$ set to the radius of the smallest sphere enclosing all the vertices of the body ( $a \approx 17.5 \mathrm{~km}$ ), 
and up to degree and order 100. In order to assess their accuracy, we ran two kinds of experiments.

First, as done previously for the simple tetrahedron, we studied the convergence of the spherical harmonic series through comparison with analytical values of the potential. The total potential was computed from its analytical formula on a set of 10000 points randomly distributed on a sphere of radius $a$. Figure 10 presents the evolution of the root mean square relative difference for this set of points, between the total potential and its series expansion at degree $n$ derived from the coefficients obtained with our algorithm - the relative difference being defined as the ratio of the absolute difference to the variance of the total potential on this set of points. The relative differences are plotted in logarithmic scale.

The series expansion converges down to a relative accuracy (in term of representation of the total potential) of $10^{-4}$ at degree 68 . Afterwards, the accuracy gets slightly worse. This increase in error for the higher degrees shows that the error control strategy we chose for this experiment can still be improved. Though, the results do not show any explicit divergence (at least up to degree 100), which tends to confirm that the chosen method for estimated ill computed coefficient is adequate.

This kind of convergence test only gives a broad evaluation of the whole series of the computed potential harmonic coefficients. In order to assess more precisely the individual coefficients, and assuming that the computation errors are mainly due to the line integral recurrences, we computed the spherical harmonic coefficients of the potential of the body through the same process, applying however for the computation of the line integrals a numerical integration procedure. Figure 11 shows the evolution of the root mean square of the relative difference between the total potential and its series expansion at degree $n$, evaluated for the same 10000 test points, similarly to Figure 10, but expressing coefficients obtained with a numerical integration of the involved line integrals.

With the numerical integration, the series expansion converges steadily towards the analytical values as degree $n$ increases. This offers an additional confirmation of the previous interpretations: the failure of the computation at high degrees is linked indeed to the recurrent scheme proposed for the computation of the line integrals.

Figure 12 presents finally a synthetic comparison between the series expansion obtained with the recurrent computation of the line integrals and the series expansion obtained with a numerical integration of the line integrals. Plotted in this figure, for each degree $n$, is the correlation coefficient between the potential at degree $n$ derived from the coefficients obtained with our algorithm (recurrent computation of the line integrals) and the potential at degree $n$ derived from the coefficients obtained though a numerical integration of the line integrals.

The recurrent computation appears to apply up to degree 62 , with a correlation with the numerical integration method greater than 0.99. Figure 13 presents a more detailed comparison between the recurrent 
scheme and the results of the numerical integration, by showing the relative difference map (in logarithmic scale) between theses two computations. There is clearly a limit around degree 60 , after which the two computations differ considerably.

\section{Concluding remarks}

The linear recursive algorithm for computing potential harmonic coefficients of constant density polyhedral sources has been revisited and expanded to the normalized case. It has been implemented and tested on a simple body. The algorithm is very efficient and works nicely for high degree and order coefficients, in contrast to the other existing recurrent algorithm known to us, which proved to be inapplicable numerically for increasing degree and order. However, the linear algorithm is connected to certain numerical instabilities emerging from the existing theoretical limitations of the linear recurrent method. Although the approach works perfectly for horizontal tetrahedral faces, it becomes unstable for every other orientation of the faces. We showed that a permanent evaluation of the appropriate rotation matrix which can link each case to the horizontal configuration can solve this problem and thus facilitate the method to be used for other applications, such as DEM-related potential quantities, airborne or satellite applications.

Acknowledgement. The present research has been carried out in the frame of France-Greek bilateral cooperation PHC Platon, project number 15063 VE. Special thanks go to Bob Werner, Jet Propulsion Laboratory, for providing us with his C code of the algorithm presented in Werner (1997). 


\section{References}

Balmino G (1994) Gravitational potential harmonics from the shape of an homogeneous body. Cel Mech Dyn Astr 60: 331-364

Barnett CT (1976) Theoretical modeling of the magnetic and gravitational fields of an arbitrary shaped three-dimensional body. Geophysics 41: 1353-1364

Chao BF, Rubincam DP (1989) The gravitational field of Phobos. Geoph Res Let 16: 859-862

Heiskanen WA, Moritz H (1967) Physical Geodesy. WH Freeman and Company, San Fransisco

Jamet O, Thomas E (2004) A linear algorithm for computing the spherical harmonic coefficients of the gravitational potential from a constant density polyhedron. In Proc Second International GOCE User Workshop, "GOCE, The Geoid and Oceanography", ESA-ESRIN, Frascati, Italy, 8-10 March 2004, ESA SP-569, June 2004

Lien SL, Kajiya JT (1984) A symbolic method for calculating the integral properties of arbitrary nonconvex polyhedra. IEEE Comp Graph Appl 4: 35-41

Martinec Z, Peck K, Bursa M (1989) The Phobos gravitational field modeled on the basis of its topography. Earth, Moon and Planets 45: 219-235

Petrović S (1996) Determination of the potential of homogeneous polyhedral bodies using line integrals, J Geod, 71: 44-52

Pohanka V (1988) Optimum expression for computation of the gravity field of a homogeneous polyhedral body. Geophys Prosp 36: 733-751

Press WH, Teukolsky SA, Vetterling WT, Flannery BP (1992) Numerical Recipes in C, the art of scientific computing. Cambridge University Press, Cambridge

Simonelli DP, Thomas PC, Carcich BT, Vererka J (1993) The generation and use of numerical shape models for irregular solar system objects. Icarus 103: 49-61

Tsoulis D (1999) Multipole expressions for the gravitational field of some finite bodies. Bollett Geod Sc Aff, 58: $353-381$ 
Tsoulis D (2001) Terrain correction computations for a densely sampled DTM in the Bavarian Alps. J Geod 75: 291-307

Tsoulis D, Petrović S (2001) On the singularities of the gravity field of a homogeneous polyhedral body. Geophysics 66: 535-539

Werner RA (1994) The gravitational potential of a homogeneous polyhedron. Cel Mech and Dyn Astr 59: $253-278$

Werner RA (1997) Spherical harmonic coefficients for the potential of a constant-density polyhedron. Comp Geosc 23: 1071-1077

Werner RA, Scheeres DJ (1996) Exterior gravitation of a polyhedron derived and compared with harmonic and mascon gravitation representations of asteroid 4769 Castalia. Cel Mech Dyn Astr 65: 313-344 


\section{Tables}

Table 1. Comparison between analytical and series expansion solutions for varying distance of the computation point with respect to the attracting source for two different maximum degrees of the respective expansion. Unit is $\mathrm{m}^{2} \mathrm{~s}^{-2}$.

\begin{tabular}{cccccc}
\hline $\begin{array}{c}\text { Distance from } \\
\text { the origin }\end{array}$ & (A) Analytic & (B) $N_{\max }=2$ & (C) $N_{\max }=10$ & $(\mathrm{~A})-(\mathrm{B})$ & $(\mathrm{A})-(\mathrm{C})$ \\
\hline 2.45 & $9.2503 \mathrm{e}-8$ & $9.3980 \mathrm{e}-8$ & $9.2528 \mathrm{e}-8$ & $-1.4768 \mathrm{e}-009$ & $-2.5253 \mathrm{e}-011$ \\
2.69 & $8.5082 \mathrm{e}-8$ & $8.6111 \mathrm{e}-8$ & $8.5092 \mathrm{e}-8$ & $-1.0292 \mathrm{e}-009$ & $-9.8991 \mathrm{e}-012$ \\
2.94 & $7.8695 \mathrm{e}-8$ & $7.9429 \mathrm{e}-8$ & $7.8699 \mathrm{e}-8$ & $-7.3325 \mathrm{e}-010$ & $-4.0011 \mathrm{e}-012$ \\
3.18 & $7.3156 \mathrm{e}-8$ & $7.3690 \mathrm{e}-8$ & $7.3158 \mathrm{e}-8$ & $-5.3343 \mathrm{e}-010$ & $-1.6853 \mathrm{e}-012$ \\
3.43 & $6.8316 \mathrm{e}-8$ & $6.8712 \mathrm{e}-8$ & $6.8317 \mathrm{e}-8$ & $-3.9568 \mathrm{e}-010$ & $-7.4149 \mathrm{e}-013$ \\
3.67 & $6.4056 \mathrm{e}-8$ & $6.4355 \mathrm{e}-8$ & $6.4056 \mathrm{e}-8$ & $-2.9877 \mathrm{e}-010$ & $-3.4049 \mathrm{e}-013$ \\
3.92 & $6.0282 \mathrm{e}-8$ & $6.0512 \mathrm{e}-8$ & $6.0283 \mathrm{e}-8$ & $-2.2930 \mathrm{e}-010$ & $-1.6282 \mathrm{e}-013$ \\
4.16 & $5.6919 \mathrm{e}-8$ & $5.7097 \mathrm{e}-8$ & $5.6919 \mathrm{e}-8$ & $-1.7860 \mathrm{e}-010$ & $-8.0857 \mathrm{e}-014$ \\
4.41 & $5.3903 \mathrm{e}-8$ & $5.4044 \mathrm{e}-8$ & $5.3903 \mathrm{e}-8$ & $-1.4099 \mathrm{e}-010$ & $-4.1581 \mathrm{e}-014$ \\
4.65 & $5.1186 \mathrm{e}-8$ & $5.1299 \mathrm{e}-8$ & $5.1186 \mathrm{e}-8$ & $-1.1267 \mathrm{e}-010$ & $-2.2083 \mathrm{e}-014$ \\
4.90 & $4.8726 \mathrm{e}-8$ & $4.8817 \mathrm{e}-8$ & $4.8726 \mathrm{e}-8$ & $-9.1047 \mathrm{e}-011$ & $-1.2081 \mathrm{e}-014$ \\
\hline
\end{tabular}

Table 2. Comparison between relative errors of truncated errors of series expansions $(A, C)$ for the tetrahedron (recursive algorithm) and (B,D) for a point mass (analytical expansion). Unit is $\mathrm{m}^{2} \mathrm{~s}^{-2}$.

\begin{tabular}{ccccc}
\hline $\begin{array}{c}\text { Distance from } \\
\text { the origin }\end{array}$ & $\begin{array}{c}\text { (A) Tetrahedron } \\
N_{\max }=2\end{array}$ & $\begin{array}{c}\text { (B) Point mass } \\
N_{\max }=2\end{array}$ & $\begin{array}{c}\text { (C) Tetrahedron } \\
N_{\max }=10\end{array}$ & $\begin{array}{c}\text { (D) Point mass } \\
N_{\max }=10\end{array}$ \\
\hline 2.45 & $1.60 \%$ & $0.75 \%$ & $0.027 \%$ & $0.015 \%$ \\
2.69 & $1.21 \%$ & $0.57 \%$ & $0.012 \%$ & $0.014 \%$ \\
2.94 & $0.93 \%$ & $0.45 \%$ & $0.005 \%$ & $0.013 \%$ \\
3.18 & $0.73 \%$ & $0.36 \%$ & $0.002 \%$ & $0.012 \%$ \\
3.43 & $0.58 \%$ & $0.29 \%$ & $0.001 \%$ & $0.011 \%$ \\
3.67 & $0.47 \%$ & $0.24 \%$ & $0.001 \%$ & $0.010 \%$ \\
3.92 & $0.38 \%$ & $0.20 \%$ & $0.000 \%$ & $0.009 \%$ \\
4.16 & $0.31 \%$ & $0.16 \%$ & $0.000 \%$ & $0.008 \%$ \\
4.41 & $0.26 \%$ & $0.14 \%$ & $0.000 \%$ & $0.008 \%$ \\
4.65 & $0.22 \%$ & $0.12 \%$ & $0.000 \%$ & $0.008 \%$ \\
4.90 & $0.19 \%$ & $0.10 \%$ & $0.000 \%$ & $0.008 \%$ \\
\hline
\end{tabular}




\section{Figure Captions}

Fig. 1. Algorithmic representation of a prismatic source through a number of simple tetrahedra. The harmonic coefficients for the potential of the prism computed at point $P$ according to the recurrent procedure of the linear algorithm will be obtained by partitioning the prism into the 6 tetrahedra shown here, all having the common vertex $P$. The final values for the coefficients will be obtained algebraically according to $U=-U_{1}-U_{2}-U_{3}+$ $U_{4}+U_{5}+U_{6}$.

Fig. 2. Coordinate systems (a) and basic geometric definitions (face and edge numbering) for the tetrahedron representation.

Fig. 3. Assessment of the recursive algorithm for the polyhedral potential harmonic coefficients for the degree range 1-10 for case study 1 vs the analytical solution (straight line).

Fig. 4. Assessment of the recursive algorithm for the polyhedral potential harmonic coefficients for the degree range 1-10 for case study 2 vs the analytical solution (straight line).

Fig. 5. Assessment of the recursive algorithm for the polyhedral potential harmonic coefficients for the degree range 10-50 for case study 2 vs the analytical solution (straight line).

Fig. 6. Effect of changing the location of the coordinate origin. The series expansion has been computed up to degree 360 .

Fig. 7. Maximum eigenvalue $\lambda$ of the recurrence matrix $A_{\infty}$ as a function of the ratio $\frac{n}{m}$ (from 1 to 10) and the position factor $\frac{\sqrt{d^{2}-o_{z}^{2}}}{a}$ (from 0 to 1 )

Fig. 8. Relative accuracy (logarithmic gray scale) of the estimates of the potential harmonic coefficients of the test tetrahedron, expressing the comparison with values obtained after perturbating the inputs of the line integral recurrence.

Fig. 9. 433 EROS - shape model consisting of 1708 faces (axes labelled in $\mathrm{km}$ )

Fig. 10. Evolution with degree of the relative differences (in logarithmic scale) between the spherical harmonics expansion at degree $\mathrm{n}$ and the analytical value of the total potential, estimated as the root mean square of a set 
of 10000 uniformly distributed random points at distance $a$ of the origin of the frame (recurrent computation of the line integrals).

Fig. 11. Evolution with degree of the relative differences (in logarithmic scale) between the spherical harmonics expansion at degree $\mathrm{n}$ and the analytical value of the total potential, estimated as the root mean square of a set of 10000 uniformly distributed random points at distance $a$ of the origin of the frame (numerical integration of the line integrals).

Fig. 12. Correlation coefficient as a function of the degree between the potential at degree $n$ derived from the computed coefficients (recurrent computation of the line integrals) and the potential at degree $n$ derived from the coefficients computed through a numerical integration of the line integrals.

Fig. 13. Decimal logarithm of the relative differences between the coefficients computed with the complete recurrent scheme, and the coefficients computed through a numerical integration of the involved line integrals. (vertically: degree $n$; horizontally: order $m$, positive orders indicate the coefficients $C_{n, m}$ and negative orders the coefficients $S_{n,-m}$ ). 


\section{Figures}
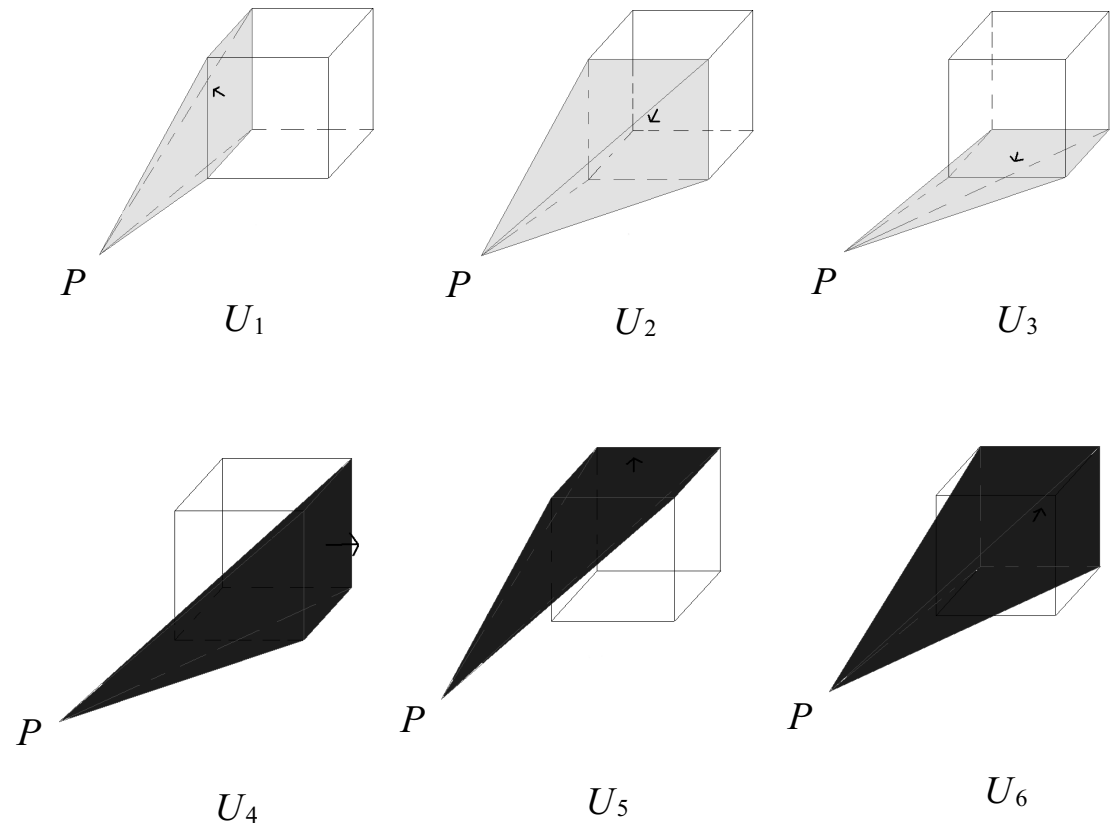

Fig. 1. Algorithmic representation of a prismatic source through a number of simple tetrahedra. The harmonic coefficients for the potential of the prism computed at point $P$ according to the recurrent procedure of the linear algorithm will be obtained by partitioning the prism into the 6 tetrahedra shown here, all having the common vertex $P$. The final values for the coefficients will be obtained algebraically according to $U=-U_{1}-U_{2}-U_{3}+$ $U_{4}+U_{5}+U_{6}$.

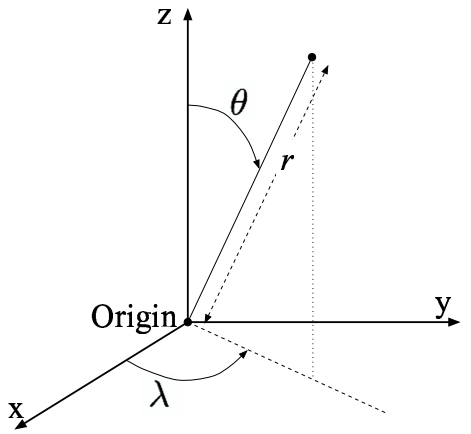

(a)

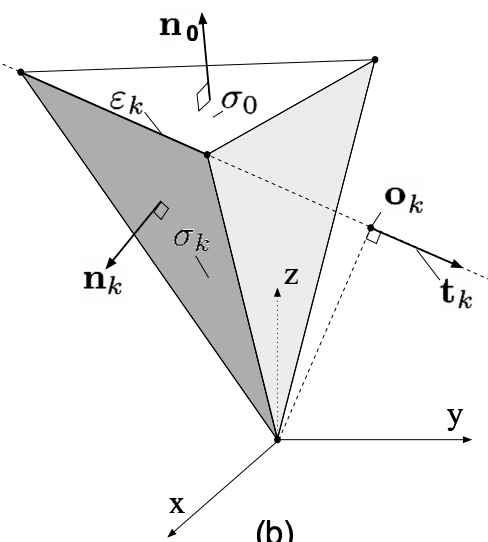

(b)

Fig. 2. Coordinate systems (a) and basic geometric definitions (face and edge numbering) for the tetrahedron representation. 


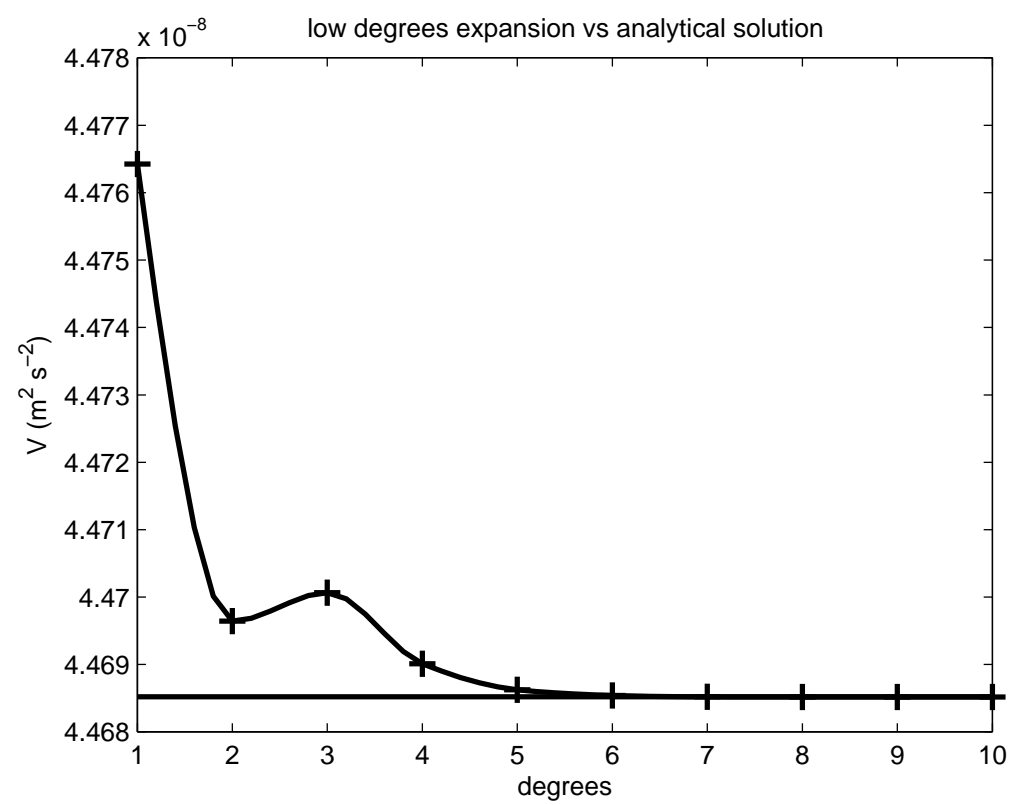

Fig. 3. Assessment of the recursive algorithm for the polyhedral potential harmonic coefficients for the degree range 1-10 for case study 1 vs the analytical solution (straight line).

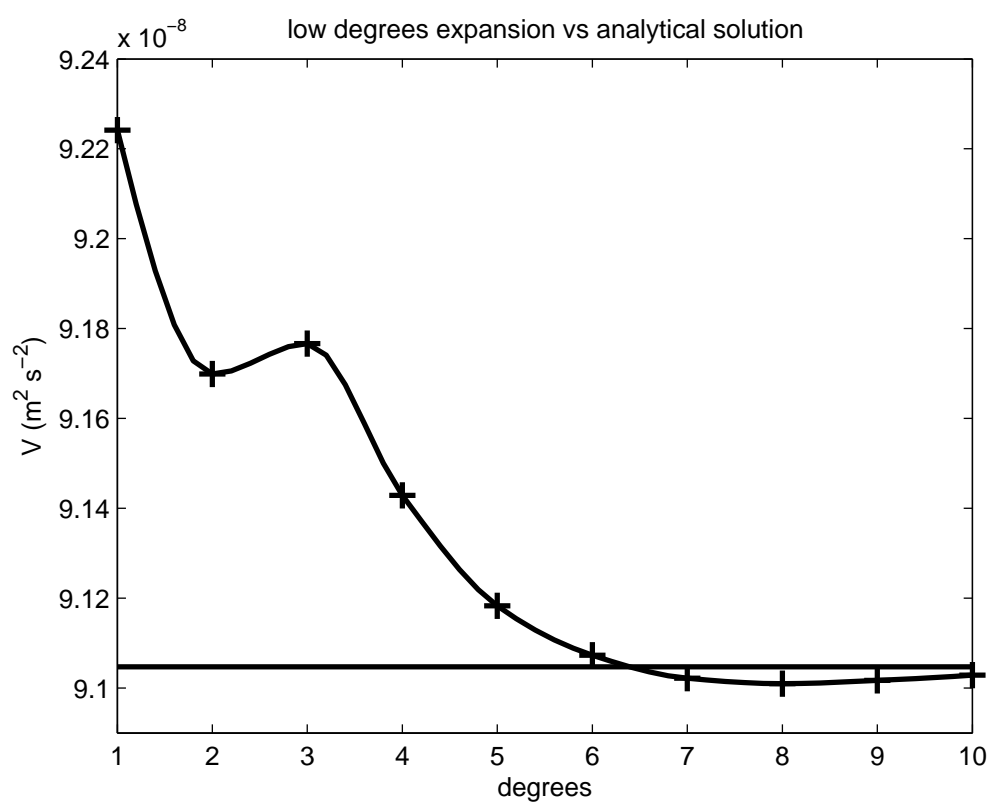

Fig. 4. Assessment of the recursive algorithm for the polyhedral potential harmonic coefficients for the degree range 1-10 for case study 2 vs the analytical solution (straight line). 


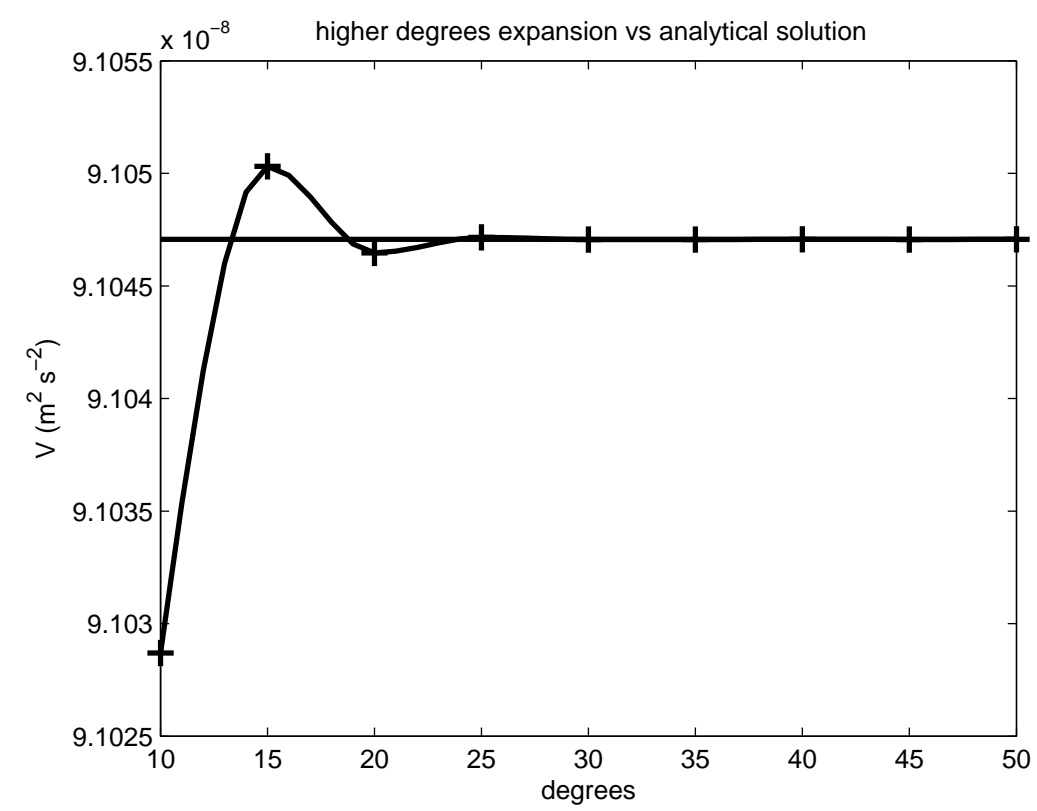

Fig. 5. Assessment of the recursive algorithm for the polyhedral potential harmonic coefficients for the degree range 10-50 for case study 2 vs the analytical solution (straight line).

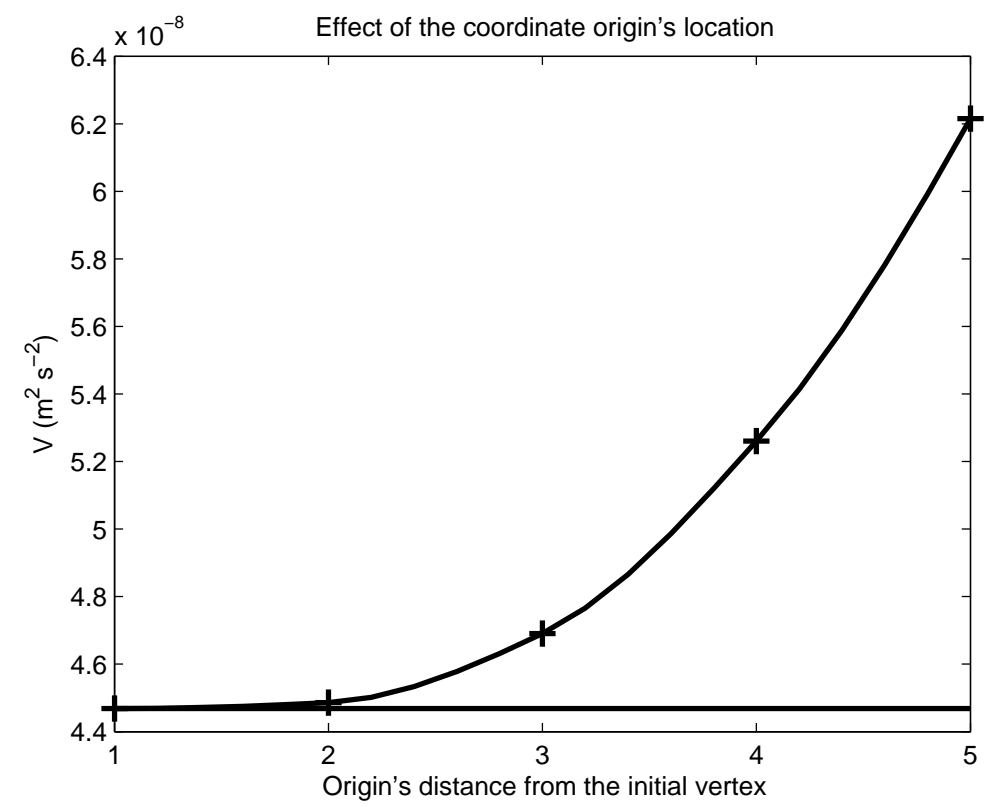

Fig. 6. Effect of changing the location of the coordinate origin. The series expansion has been computed up to degree 360 . 


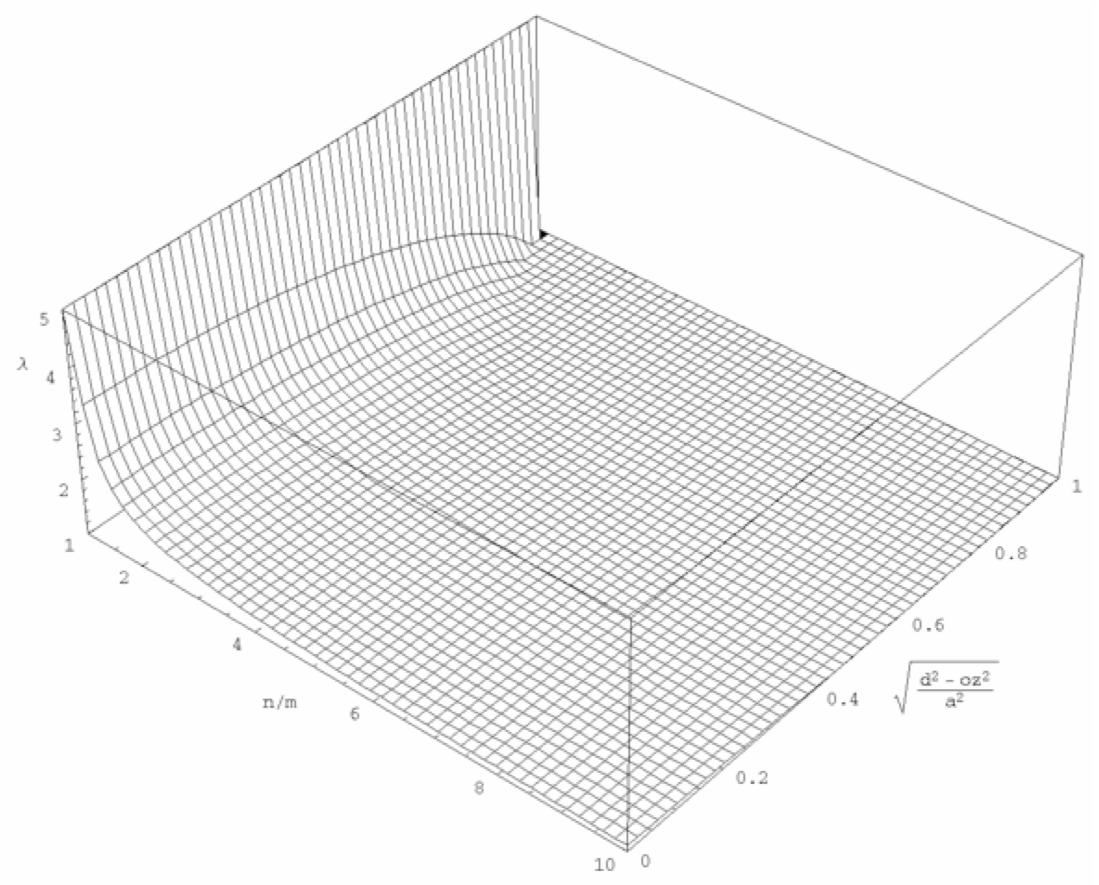

Fig. 7. Maximum eigenvalue $\lambda$ of the recurrence matrix $A_{\infty}$ as a function of the ratio $\frac{n}{m}$ (from 1 to 10 ) and the position factor $\frac{\sqrt{d^{2}-o_{z}^{2}}}{a}$ (from 0 to 1 )

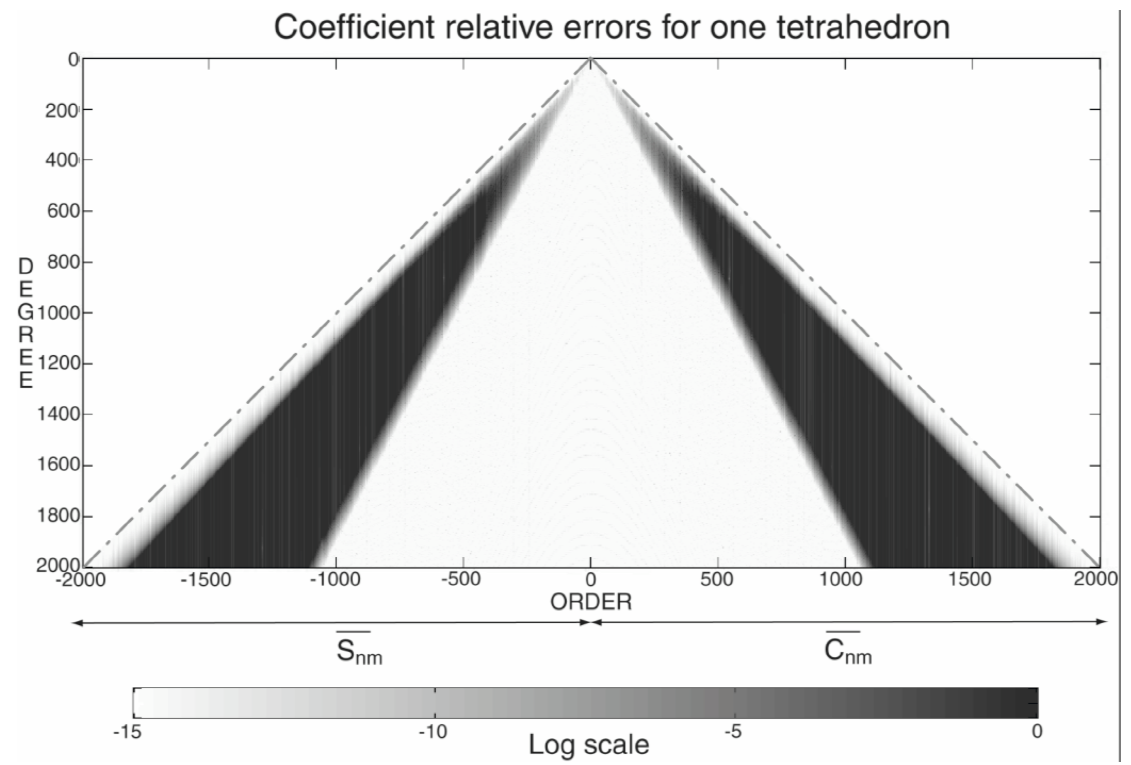

Fig. 8. Relative accuracy (logarithmic gray scale) of the estimates of the potential harmonic coefficients of the test tetrahedron, expressing the comparison with values obtained after perturbating the inputs of the line integral recurrence. 


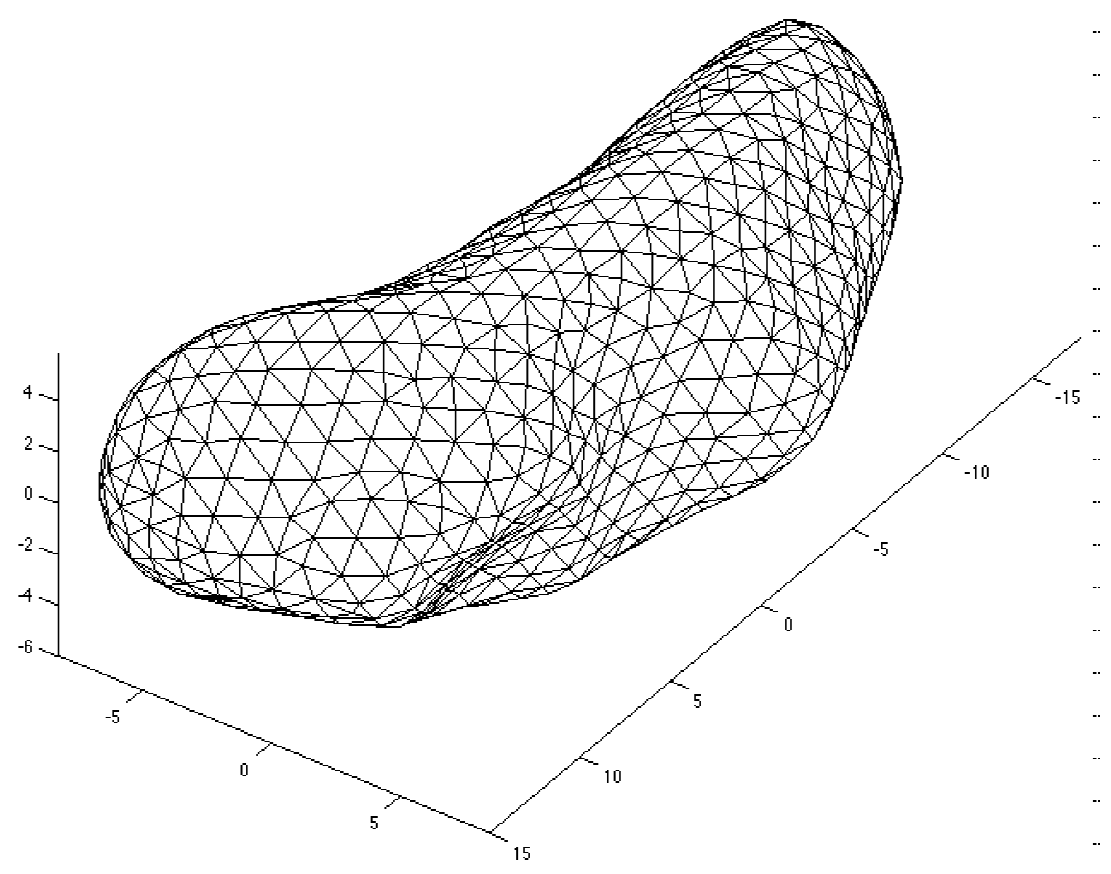

Fig. 9. 433 EROS - shape model consisting of 1708 faces (axes labelled in $\mathrm{km}$ )

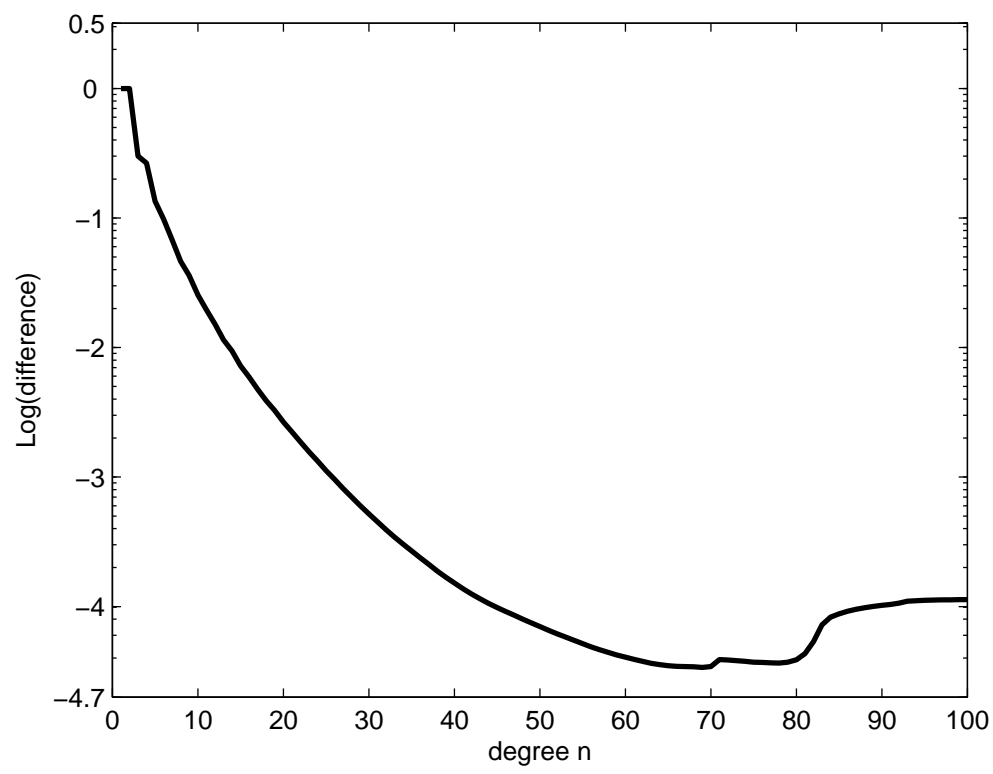

Fig. 10. Evolution with degree of the relative differences (in logarithmic scale) between the spherical harmonics expansion at degree $\mathrm{n}$ and the analytical value of the total potential, estimated as the root mean square of a set of 10000 uniformly distributed random points at distance $a$ of the origin of the frame (recurrent computation of the line integrals). 


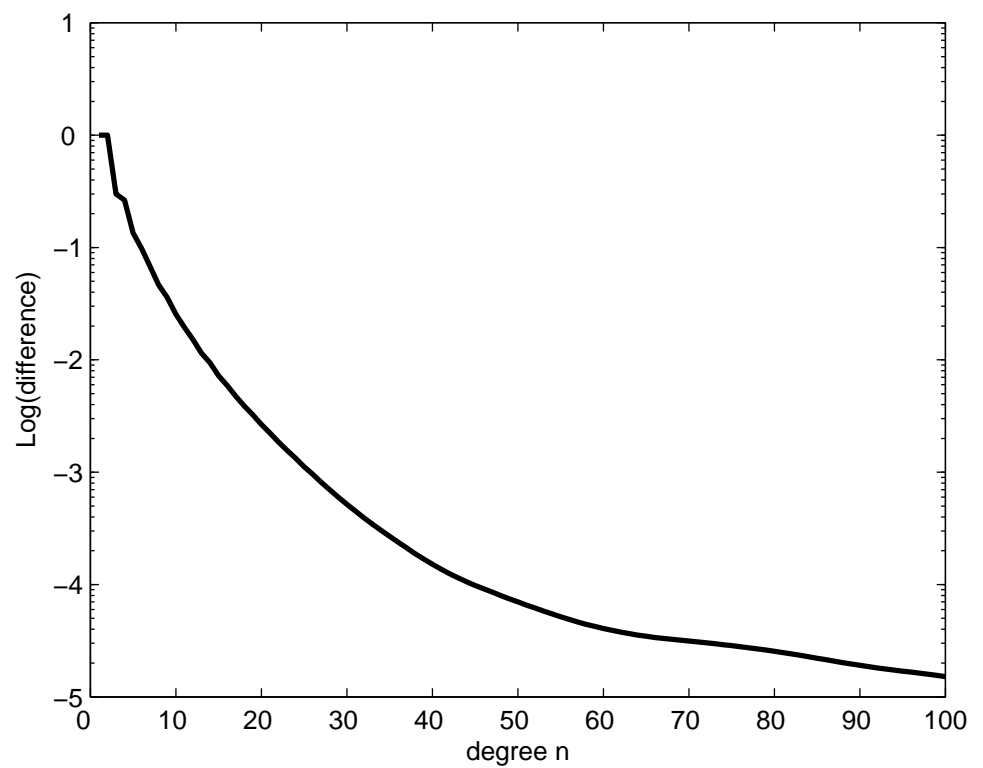

Fig. 11. Evolution with degree of the relative differences (in logarithmic scale) between the spherical harmonics expansion at degree $\mathrm{n}$ and the analytical value of the total potential, estimated as the root mean square of a set of 10000 uniformly distributed random points at distance $a$ of the origin of the frame (numerical integration of the line integrals). 


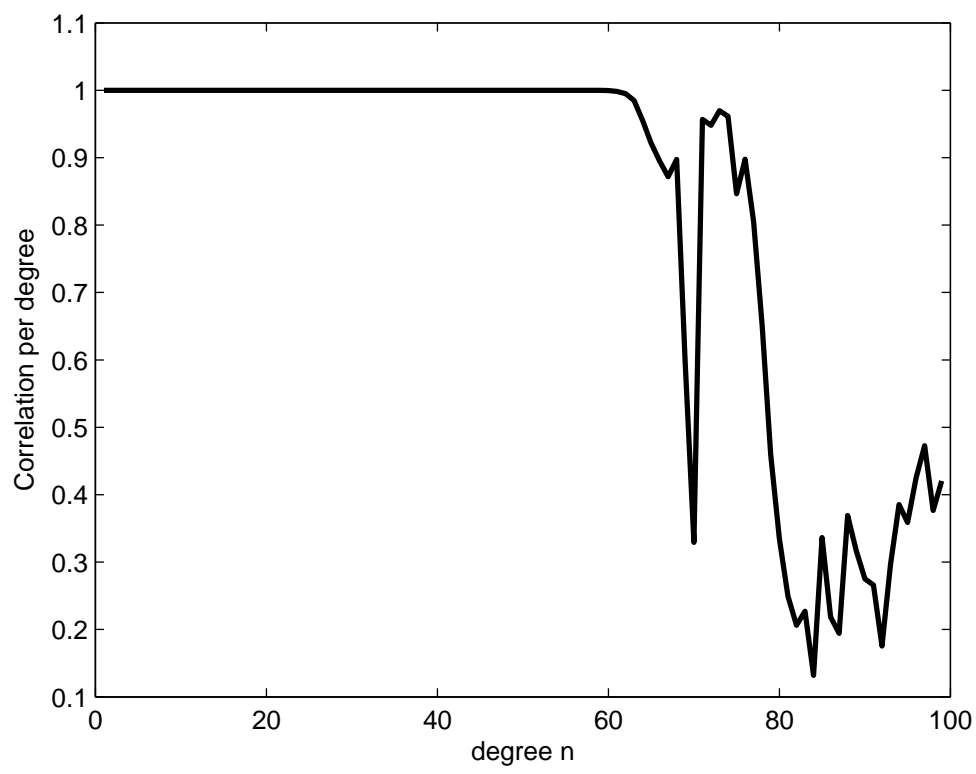

Fig. 12. Correlation coefficient as a function of the degree between the potential at degree $n$ derived from the computed coefficients (recurrent computation of the line integrals) and the potential at degree $n$ derived from the coefficients computed through a numerical integration of the line integrals.

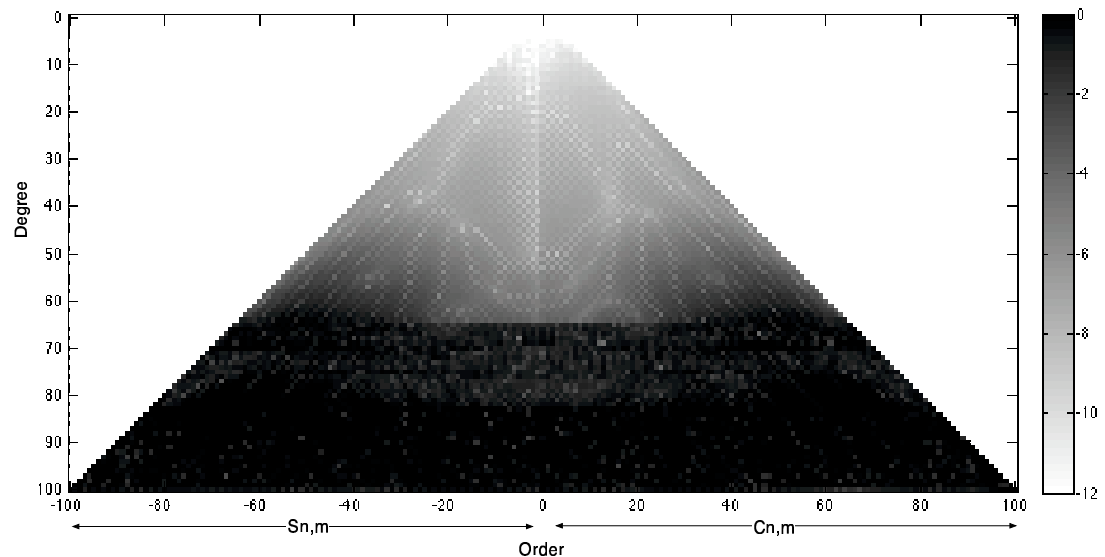

Fig. 13. Decimal logarithm of the relative differences between the coefficients computed with the complete recurrent scheme, and the coefficients computed through a numerical integration of the involved line integrals. (vertically: degree $n$; horizontally: order $m$, positive orders indicate the coefficients $C_{n, m}$ and negative orders the coefficients $S_{n,-m}$ ). 